\title{
Perceptual effects of unequal saccadic adaptation produced by a dichoptic step
}

\section{Anna Kosovicheva}

\author{
Peter J. Bex
}

\author{
Department of Psychology, Northeastern University, \\ Boston, MA, USA
}

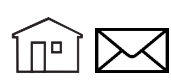

Department of Psychology, Northeastern University, Boston, MA, USA

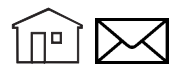

The binocular coordination of eye movements in a three-dimensional environment involves a combination of saccade and vergence movements. To maintain binocular accuracy and control in the face of sensory and motor changes (that occur with e.g., normal aging, surgery, corrective lenses), the oculomotor system must adapt in response to manifest visual errors. This may be achieved through a combination of binocular and monocular mechanisms, including the recalibration of saccade and vergence amplitudes in response to different visual errors induced in each eye (Maiello, Harrison, \& Bex, 2016). This work has used a double-step paradigm to recalibrate eye movements in response to visual errors produced by dichoptic target steps (e.g., leftward in the left eye and rightward in the right eye). Here, we evaluated the immediate perceptual effects of this adaptation. Experiment 1 measured localization errors following adaptation by comparing the apparent locations of pre- and postsaccadic probes. Consistent with previous work showing localization errors following saccadic adaptation, our results demonstrated that adaptation to a dichoptic step produces different localization errors in the two eyes. Furthermore, in Experiment 2, this effect was reduced for a vergence shift in the absence of a saccade, indicating that saccade programming is responsible for a large component of this illusory shift. Experiment 3 measured postsaccadic stereopsis thresholds and indicated that, unlike localization judgments, adaptation did not influence stereoacuity. Together, these results demonstrate novel dichoptic visual errors following oculomotor adaptation and point to monocular and binocular mechanisms involved in the maintenance of binocular coordination. directed to objects at different depth planes that require slightly different eye movements in each eye. Although this binocular coordination in three-dimensional space is critical for many everyday tasks, the underlying mechanism has historically been debated. In the 19th century, Hering (1868) proposed that control of the two eyes originates from a shared neural signal, and that a movement in one eye is accompanied by an equal amplitude movement (in the same or opposite direction) in the other eye. Thus any eye movement can be described as the superposition of conjugate and vergence components that are equal in magnitude between the two eyes. In contrast, Helmholtz (1867) argued that the eyes are innervated independently, and that binocular coordination is instead achieved through learning. Over the last several decades, neurophysiological and eye tracking evidence has accumulated on both sides of this debate (for reviews, see Coubard, 2013; King, 2011). One possible reconciliation for these conflicting findings is that binocular coordination may be accomplished through a combination of monocular and binocular mechanisms (Coubard, 2013).

Regardless of the underlying mechanisms, to maintain accuracy and binocular control, the two eyes must be able to respond appropriately to visual errors. Errors in eye movements can arise from uncertainty or imprecision inherent in the visual and oculomotor systems, or more systematically as a result of changes to visual input (e.g., corrective lenses) or anatomic changes concerning the eyes (e.g., surgery, trauma, or changes that occur with normal aging). Much of the experimental literature has examined recalibration over short timescales, measuring oculomotor responses to systematic visual errors. An example of this is the adaptation of saccadic eye movement amplitudes (for reviews, see Hopp \& Fuchs, 2004; Pélisson, Alahyane, Panouillères, \& Tilikete, 2010). A typical saccadic adaptation experiment uses a double-step paradigm (McLaughlin, 1967), in which the observer saccades from a fixation point to a target (first step),

To enable us to successfully interact with our environment, the two eyes generally move in a coordinated manner, bringing objects of interest to the fovea of each eye. Many of these refixations are Citation: Kosovicheva, A., \& Bex, P. J. (2020). Perceptual effects of unequal saccadic adaptation produced by a dichoptic step. Journal of Vision, 20(5):7, 1-21, https://doi.org/10.1167/jov.20.5.7. 
and at the moment that the saccade is initiated, the target undergoes a secondary shift in location. This second step is generally not observed due to saccadic suppression (e.g., Bridgeman, Hendry, \& Stark, 1975; Volkmann, 1962), and produces an apparent overshoot or undershoot on saccade completion. Initially, the observer makes a corrective saccade, and then, over dozens of trials, saccade amplitudes are recalibrated to minimize this visual error. This robust phenomenon can be measured reliably with a small number of observers and is normally induced within 50 to 100 saccades in humans (Deubel, Wolf, \& Hauske, 1986). Saccadic adaptation has most often been tested with monocular targets (e.g., McLaughlin, 1967), or with identical target shifts in the two eyes (e.g., Deubel et al., 1986).

More recently - and relevant to the debate between Hering and Helmholtz - saccadic adaptation has also been demonstrated for dichoptic target steps (Maiello, Harrison, \& Bex, 2016). This paradigm is similar to a standard saccadic adaptation experiment, with the target shifted in opposite directions in the two eyes (e.g., leftward in the left eye and rightward in the right eye) on saccade onset. Initially, this shift produces a vergence movement that begins after saccade completion. After a few dozen trials, there are changes in both the saccade and vergence components: in this example, saccade amplitudes decrease in the left eye and increase in the right eye, whereas the vergence movement decreases in amplitude and in latency. The interocular difference in saccade gain provides support for monocular mechanisms, suggesting that there may be some degree of independent oculomotor control in the two eyes. Similar work has demonstrated that observers can produce disconjugate saccades following adaptation to a prism-induced disparity (Averbuch-Heller, Lewis, \& Zee, 1999) or adaptation to anisometropic corrective lenses (Erkelens, Collewijn, \& Steinman, 1989; Lemij \& Collewijn, 1991). At the same time, binocular mechanisms are an important component of saccadic adaptation. For example, Albano and Marrero (1995) showed interocular transfer of saccadic adaptation; when one eye is occluded during adaptation, the training transfers from the unoccluded eye to the occluded eye. An account of saccadic adaptation involving a combination of monocular and binocular mechanisms operating at different levels could reconcile these different results, and would be consistent with evidence that adaptation is distributed across multiple neural loci in humans (Hopp \& Fuchs, 2004).

More broadly, these results demonstrate that some component of binocular control is adaptable and may be altered through learning, at least partly consistent with Helmholtz's original proposal. In principle, this mechanism could be responsible for changes that occur over the lifespan. For example, normal aging can produce displacement of the extraocular muscles
(Clark \& Demer, 2002), and changes in the size and shape of the eye sockets (Shaw et al., 2011). In other instances, changes in oculomotor control may occur in response to trauma or surgery. One possibility is that saccadic adaptation in response to anatomic changes may rely on similar mechanisms as those observed during short-term saccadic adaptation using a double-step paradigm. For example, in nonhuman primates, surgical weakening of the eye muscles produces adaptation similar to that produced by a visual target displacement when the two are measured under comparable circumstances (Scudder, Batourina, \& Tunder, 1998). An understanding of monocular and binocular contributions to saccadic adaptation in the laboratory may therefore inform research on the development of binocular control in children, as well as changes that occur in aging populations. It would also help to determine the capability of similar procedures to improve oculomotor control in individuals with strabismus or vergence insufficiencies.

Although previous work has demonstrated that the oculomotor system can recalibrate saccade amplitudes unequally in response to dichoptic target steps (Maiello et al., 2016), the implications for sensory processing are presently unknown. An understanding of the perceptual consequences of adaptation can help to differentiate between different functional levels of adaptation, and whether adaptive changes occur at an early stage that influences both sensory and motor processes (see Pélisson et al., 2010 for a review). In addition, if similar mechanisms are involved in adaptation to long-term changes in sensory input, in response to corrective lenses or anatomic changes (e.g., surgery, trauma, or normal aging) as outlined earlier, it is important to understand how these changes might influence perception. Using procedures similar to Maiello et al. (2016), we tested the influence of disconjugate changes in saccades on two different perceptual tasks: localization with monocular targets (Experiments 1 and 2 ), and binocular depth discrimination (Experiment 3 ). Together, these experiments test whether perception simultaneously compensates for changes in saccade amplitude that are different between the two eyes. Previous work has indicated that adaptive changes in saccades are tied to changes in visual localization (e.g., Awater, Burr, Lappe, Morrone, \& Goldberg, 2005; Bahcall \& Kowler, 1999; Collins, Rolfs, Deubel, \& Cavanagh, 2009) and motion discrimination thresholds (Mack, Fendrich, \& Pleune, 1978). To the extent that conjugate and disconjugate adaptive changes rely on shared neural mechanisms, we predict that adaptation would alter perception in a similar manner. However, another possibility is that disconjugate changes rely on a separate set of neural mechanisms. For example, previous work has proposed separable mechanisms for different directional changes (i.e., amplitude increases vs. decreases; Panouillères et al., 2009), and different 


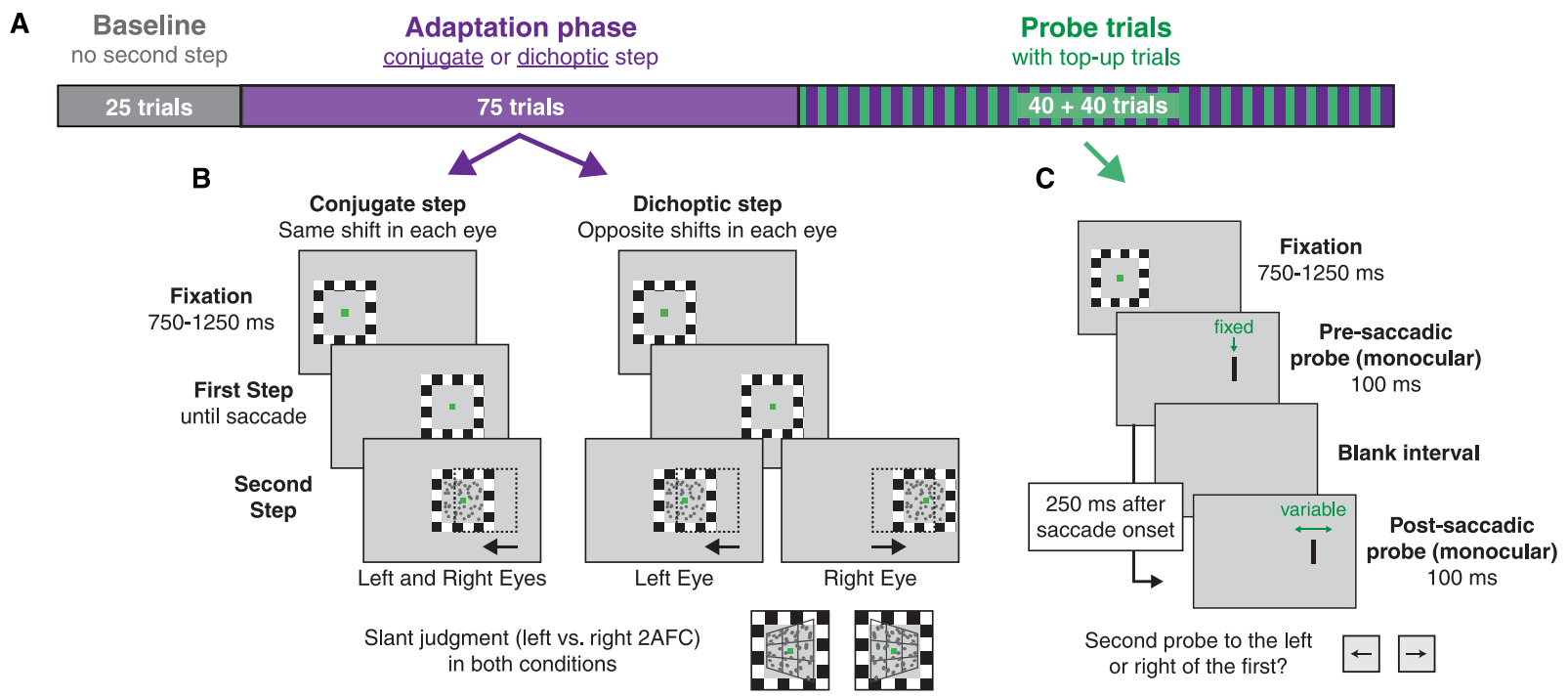

Figure 1. Stimuli and procedure for Experiment 1. (A) Sequence of trial types within each block. Observers completed eight blocks of trials, each consisting of 25 baseline trials, followed by 75 adaptation trials, then 80 probe localization trials that alternated with top-up adaptation trials (40 trials of each). (B) Sequence of events for adaptation and top-up adaptation trials. Observers fixated the center of the target, which then shifted $8^{\circ}$ to the right (first step). Once the observer initiated a saccade to the target, it stepped a second time, based on one of two adaptation conditions. The second step was either a conjugate step, which had the same shift in both eyes, or a dichoptic step, which shifted in opposite directions in the two eyes. On saccade completion, observers performed a 2AFC slant judgment on a set of random dots inside the frame (step sizes and stimuli not to scale). (C) Sequence of events in probe localization trials. Following an initial fixation interval, observers were shown a briefly flashed $(100 \mathrm{~ms})$ line $8^{\circ}$ to the right of fixation and were instructed to saccade to the probe, which disappeared before saccade completion. At $250 \mathrm{~ms}$ after saccade onset, a second probe line was flashed at one of five different locations, and observers judged whether the second line (the test line) was to the left or to the right of the first line (the reference). The probes were monocular, and always shown to the same eye within a given trial. The text shown above the probes illustrates the probe locations and was not displayed to subjects.

types of saccades (e.g., voluntary vs. involuntary; Alahyane et al., 2007).

To distinguish between these hypotheses, it is necessary to first demonstrate unequal saccadic adaptation between the two eyes using a dichoptic target step (Figure 1), as previously shown by Maiello et al. (2016). Based on this previous work, we predict that this adaptation would manifest as a difference in gain changes between the two eyes, and as a change in saccade amplitude from baseline measurements (Figure 2). Second, to measure its perceptual effects, it is necessary to establish that adaptation is retained in some way following a disconjugate saccade. To effectively test the perceptual effects of adaptation with intrasaccadic or postsaccadic targets, the eyes need to stay diverged over the immediate postsaccadic window (i.e., roughly 50-500 ms after saccade onset). We therefore expect to see nonzero, sustained divergence during probe trials after saccades are adapted (Figure 3). In Experiment 1, we measured the degree to which adaptation is retained, as well as the immediate effects of adaptation on position judgments. We then performed a second experiment, repeating the same procedures with a vergence movement but not a saccade, to measure the relative contributions of saccadic and vergence mechanisms to adaptation. In a third and final experiment, we tested the immediate effects of adaptation on stereoacuity.

\section{Experiment 1: Localization errors}

Previous work has demonstrated that saccadic adaptation has important perceptual consequences. For example, following a period of adaptation to a double-step stimulus, Bahcall and Kowler (1999) instructed observers to compare the perceived locations of briefly flashed pre- and postsaccadic probes. Their results demonstrated an illusory mislocalization, such that postsaccadic probes needed to be shifted in the same direction as the adapting step to appear in the same location as the presaccadic probe. Other studies have reported adaptation-induced localization errors for single targets flashed during fixation that occur prior to a saccade (Awater et al., 2005; Georg \& Lappe, 2009), or in the absence of any saccade (Moidell \& Bedell, 1988; Zimmermann \& Lappe, 2010). In addition, the focus of spatial compression, normally observed during saccades (Ross, Morrone, \& Burr, 1997), is shifted 
toward the endpoint of the executed saccade, rather than the originally intended saccade (Awater et al., 2005).

Previous findings demonstrating trans-saccadic localization errors (Bahcall \& Kowler, 1999) were originally interpreted as evidence that the efference copy signal, which is used to maintain perceptual stability across saccades, is based on the originally intended saccade, rather than the executed (i.e., adapted) saccade. However, another possibility is that the efference copy is based on the executed saccade, but instead, the perceived location of the target is shifted due to adaptation (Collins et al., 2009). In other words, the efference copy signal would include the adaptive changes in saccade gain, and localization errors would be observed due to shifts in the perceived location of the presaccadic target. An advantage of this interpretation is that it is consistent with a number of other findings, including the mislocalization of probes flashed well before saccade onset (Awater et al., 2005), and similar localization errors measured across different response modalities (Bruno \& Morrone, 2007).

However, the perceptual effects of saccadic adaptation in response to a dichoptic step have not been previously measured, and it is unknown whether similar errors occur when saccade amplitudes are recalibrated in different directions in the two eyes. In principle, shifts in the perceived presaccadic target location could occur in opposite directions in the two eyes. Based on more recent interpretations of trans-saccadic localization errors (Collins et al., 2009), this result would suggest a modification or remapping of perceptual space in three dimensions, and support the existence of separate maps for oculomotor control in the two eyes. However, as discussed previously, saccadic adaptation may depend on a combination of monocular and binocular mechanisms, and may be distributed across multiple neural loci in humans. Therefore, another possibility is that trans-saccadic localization would rely on a shared neural signal between the two eyes, which would result in similar localization errors between the two eyes. Furthermore, if adaptation to a dichoptic step relies on different mechanisms than conjugate changes in saccade amplitude, localization reports following adaptation may be veridical.

To distinguish between these possibilities, in Experiment 1, we measured perceived location using similar procedures to those used by Bahcall and Kowler (1999) and Collins et al. (2009), which are outlined in Figure 1. Following a baseline period, which had no adapting step, subjects adapted in response to opposite target steps in each eye (leftward in the left eye, rightward in the right eye). After adaptation, observers compared the positions of pre- and postsaccadic probes, which were both monocular, and always presented within the same eye on a given trial. The presaccadic probe (the reference line) was fixed in location, and we varied the position of the postsaccadic probe (the test line) to determine the position at which it appeared to be in the same location as the reference. If the adaptive oculomotor changes do not influence perceived location, judgments would be veridical - the postsaccadic probe would need to be in the same location as the presaccadic probe to appear in the same position. Conversely, if we observe similar changes in localization to previous reports (Bahcall \& Kowler, 1999; Collins et al., 2009), the postsaccadic probe would appear in the same location as the presaccadic probe only if it is shifted in the direction of the adapting step - in this instance, in opposite directions in the left- and right-eye probe conditions. We compared observers' responses to those in a conjugate step control condition, in which the second step was identical in the two eyes. In addition, the use of brief monocular probes also allowed us to measure the magnitude of retention of adaptation in the absence of visual feedback: following adaptation, how much do the eyes diverge if there is no visual information to drive vergence movements?

\section{Methods}

\section{Participants}

Six observers (three women; mean age, 26.2; age range, 18-31), including one author (AK) participated in Experiment 1. All observers had normal or corrected-to-normal vision and stereoacuity of 60 arcseconds or better, measured with the Titmus stereo test. Procedures were approved by the institutional review board at Northeastern University and followed the tenets of the Declaration of Helsinki (aside from preregistration). All participants gave written informed consent prior to participating in the experiment and all observers (except the author) were naive to the purpose of the experiment.

\section{Eye tracking}

Eye movements were recorded with an Eyelink 1000 desktop mounted infrared eye tracker (SR Research Ltd., Mississauga, Ontario, Canada), used in conjunction with the Eyelink Toolbox for MATLAB (The Mathworks, Inc., Natick, MA; Cornelissen, Peters, \& Palmer, 2002). Observers were calibrated with a standard nine-point calibration procedure at the beginning of each block of trials (Stampe, 1993), while viewing the targets binocularly through the same shutter glasses used in the experiment (see Stimuli and procedure). The average error on validation was $0.56^{\circ} \pm 0.27^{\circ}$ (mean $\pm S D$ ) across observers. Gaze position was recorded binocularly at a sampling rate of $500 \mathrm{~Hz}$. Online gaze-contingent control was based 
on the average of the recorded gaze positions from the two eyes. Noise artifacts were reduced using Eyelink software, which applied a heuristic filtering algorithm to the raw gaze position samples (see Stampe, 1993, for details). For offline analysis, gaze data were classified using the Eyelink algorithm into saccades and fixations using velocity and acceleration thresholds of $30 \%$ and $8000^{\circ} / \mathrm{s}^{2}$, respectively, consistent with Maiello et al. (2016).

\section{Stimuli and procedure}

Stimuli were presented on a gamma-corrected 27-in. BenQ XL2720Z LCD monitor (BenQ Corporation, Taipei, Taiwan) controlled by a Dell Optiplex 9020 desktop computer (Dell Inc., Round Rock, TX) with a Quadro K420 graphics card (Nvidia Corporation, Santa Clara, CA). The experiment was programmed in MATLAB using the Psychophysics Toolbox Version 3 (Brainard, 1997; Kleiner, Brainard, \& Pelli, 2007; Pelli, 1997). Display resolution was set to $1920 \times 1080$, and the refresh rate to $120 \mathrm{~Hz}$. Observers viewed the display binocularly through LCD active shutter glasses synchronized to the refresh of the monitor (Nvidia 3D Vision; $60 \mathrm{~Hz}$ monocular refresh). Observers were seated at a viewing distance of $50 \mathrm{~cm}$ from the display, with head position stabilized using a chinrest. At this distance, the display subtended $67.2^{\circ}$ horizontally and $38.1^{\circ}$ vertically. All stimuli were drawn on a gray (61.4 $\mathrm{cd} / \mathrm{m}^{2}$ ) background.

Observers completed 1440 trials each, which were divided into eight blocks of 180 trials, each lasting approximately 10 to 15 minutes. Observers completed the conjugate and dichoptic step conditions in separate blocks, which alternated (A-B-A-B) over the set of eight blocks. Half of the observers started with the conjugate step condition, and the other half started with the dichoptic step condition. As shown in Figure 1A, within each block, observers completed 25 baseline trials, which had no second step, followed by 75 adaptation trials (conjugate or dichoptic step), and then 80 trials that alternated between probe trials and top-up adaptation trials. On probe trials, observers compared the position of a briefly flashed monocular probe (a vertical line) before and after the saccade. Top-up adaptation trials, which repeated the same adaptation condition, were inserted on every other trial in an effort to maintain the maximum level of adaptation while these perceptual effects were measured. Observers were calibrated at the beginning of each block, and drift correction was performed every 25 trials. Prior to starting the experiment, observers completed a short practice block consisting of 25 baseline trials and 10 probe trials, which were not included in the analysis. Each trial type is described in detail in the following sections.

\section{Baseline and adaptation trials}

Figure $1 \mathrm{~B}$ outlines the procedure for the baseline and adaptation trials. At the beginning of each trial, observers were shown a square frame (full width and height of $4^{\circ}$; line width of $0.5^{\circ}$ ) centered $4^{\circ}$ to the left of the display center. The frame was shown binocularly and consisted of alternating white $\left(142 \mathrm{~cd} / \mathrm{m}^{2}\right)$ and black $\left(\sim 0 \mathrm{~cd} / \mathrm{m}^{2}\right)$ segments $\left(0.75^{\circ} \times 0.5^{\circ} ; 0.5^{\circ}\right.$ square at the corners). Observers were instructed to fixate a small $0.2^{\circ}$ square that was centered inside the frame. The program required continuous fixation within $\pm 1.5^{\circ}$ horizontally and vertically of this fixation point for a randomly selected interval between 750 and $1250 \mathrm{~ms}$ before proceeding. The saccade target (i.e., the frame and fixation point together) then immediately shifted $8^{\circ}$ to the right (first step), and observers were instructed to saccade to this target as quickly as possible. On baseline trials, the frame remained in place after the saccade. On adaptation trials, a second step was introduced on saccade onset. Once the eyes had left the $\pm 1.5^{\circ}$ fixation region centered on the fixation point, the frame then shifted either $0.8^{\circ}$ leftward in both eyes (conjugate step condition) or $0.8^{\circ}$ leftward in the left eye and $0.8^{\circ}$ rightward in the right eye (dichoptic step condition).

On completion of the saccade, observers were instructed to fixate the small square in the center of the frame. To ensure that observers maintain stable vergence at the correct depth of the target in both adaptation conditions and to maintain participants' alertness, observers completed a two-alternative forced-choice (2AFC) stereoscopic slant judgment across both baseline and adaptation trials. The onset of the saccade triggered the appearance of a set of 125 small black dots $\left(0.1^{\circ}\right.$ diameter) randomly positioned within the inner border of the frame (a $3^{\circ}$ square region). Disparity was introduced by modifying the relative horizontal positions of the dots inside the square according to the formula: $x_{1}=s^{*} x_{2}$, where $s$ represents the slope, and $x_{1}$ and $x_{2}$ represent the horizontal positions of the dots in the two eyes, relative to the center of the frame. The assignment of $x_{1}$ and $x_{2}$ to the left and right eyes was counterbalanced across trials. To keep the dots within the bounds of the square, $x_{2}$ ranged from $-1.5^{\circ}$ to $+1.5^{\circ}$, and $s$ was always less than or equal to 1 . As this scaling factor shifted the horizontal positions of the dots relative to the center of the square, on average, there was no net disparity across the full set of dots. In other words, on half the trials, the left side of the dots had crossed disparity and the right side had uncrossed disparity, and the other half of the trials had the opposite configuration. In the dichoptic step condition, this disparity was added to the uncrossed disparity introduced by the opposite target steps $\left(1.6^{\circ}\right.$, produced by opposite shifts of $0.8^{\circ}$ in the two eyes). Therefore, to perform the task effectively in this condition, observers needed to diverge their eyes 
to the correct depth of the frame. Across all adaptation trials, observers were instructed to maintain fusion of the frame at all times, making vergence movements when necessary. Following the saccade, the program required continuous fixation within $\pm 1.5^{\circ}$ of the central fixation square for a randomly chosen interval between 750 and $1250 \mathrm{~ms}$ before disappearing. Observers were then instructed to report the perceived slant, reporting whether the left or right side appeared slanted backward in a $2 \mathrm{AFC}$ task. Responses were recorded via button press (left or right arrow key) on a keyboard. The color of the square provided feedback on the accuracy of the observer's response on the previous trial. The square was green $\left(102.3 \mathrm{~cd} / \mathrm{m}^{2}\right)$ if the response on the previous trial was correct, and red $\left(31.3 \mathrm{~cd} / \mathrm{m}^{2}\right)$ if it was incorrect.

To maintain a consistent level of difficulty in the stereoscopic slant task, the slant $(s)$ was adaptively controlled across trials using two randomly interleaved three-up, one-down staircases. The staircases ran continuously from the baseline to the adaptation and top-up adaptation trials (70 trials per staircase, 140 trials total), but started over at the beginning of each block. Each staircase had an initial value of $s$ of 0.97 and a step size of 0.005 , which correspond to approximately 162 and 27 arcseconds at the largest disparity (i.e., the disparity at the left and right sides of the cluster of dots). The step size was reduced by $25 \%$ every three reversals. The program constrained the staircase values such that the value of $s$ could not fall outside the range of 0.9 to 1 (540 and 0 arcseconds at the largest disparity, respectively).

\section{Probe trials}

Figure 1C outlines the procedure for probe trials. Observers were shown an empty frame and fixation point, centered $4^{\circ}$ to the left of screen center. Before proceeding, the program required continuous fixation within $\pm 1.5^{\circ}$ horizontally and vertically of this fixation point for a randomly selected duration between 750 and $1250 \mathrm{~ms}$. Following this interval, a monocular probe, referred to as the reference (vertical line; $1.5^{\circ}$ height and $0.25^{\circ}$ line width) was flashed at $8^{\circ}$ to the right of fixation for $100 \mathrm{~ms}$. Observers were instructed to saccade to this target and keep their eyes at that side of the screen (as the target disappeared before saccade completion). At $250 \mathrm{~ms}$ following saccade onset (defined as the eyes leaving the $\pm 1.5^{\circ}$ region around fixation), a second line (referred to as the test line) was shown at one of five horizontal positions relative to the first (reference) line, in linearly spaced steps from $-3^{\circ}$ (to the left of the first line) to $+2^{\circ}$ (to the right of the first line). The times of probe onset and offset were selected to follow previously reported procedures (Bahcall \& Kowler, 1999; Collins et al., 2009). However, in our experiment, the probes were monocular on each trial. Both lines were always presented to the same eye (either both in the left eye or both in the right eye, on separate trials). This allowed measurement of localization errors (i.e., the difference in perceived location between preand postsaccadic targets) in the left and right eyes separately. On each probe trial, observers reported (via button press) whether the second line appeared to the left or right of the first line in a $2 \mathrm{AFC}$ task. Each block had four probe trials for each unique combination of postsaccadic target position and eye condition, which were randomly interleaved, for a total of 40 (five locations $\times$ two eyes $\times$ four repeats) probe trials per block, and 320 probe trials across the eight blocks. As we expected localization judgments to reflect illusory shifts, as reported in Bahcall and Kowler (1999) and Collins et al. (2009), observers were not given any feedback on the accuracy of their responses on the probe trials. The fixation square on the next trial was white to indicate an absence of feedback.

\section{Data analyses}

\section{Eye tracking data}

The analysis procedures were similar to those reported by Maiello et al. (2016). Gaze recordings were analyzed two ways: (1) measurements of saccade amplitude as a function of trial number (Figure 2A), similar to typical saccadic adaptation experiments (Deubel et al., 1986); and (2) measurements of interocular divergence relative to saccade onset (Figure 3A). This second analysis allows us to quantify the postsaccadic vergence component of the adaptation effect and measure any retention during probe trials.

For the analysis of saccade amplitudes, for each trial, we analyzed the first saccade following stimulus onset that was between $5^{\circ}$ and $11^{\circ}$ in amplitude, calculated as the difference in horizontal position between the end and start of the saccade. Trials that had saccade amplitudes outside these bounds, or that had saccade onset latencies lower than $80 \mathrm{~ms}$ or greater than $500 \mathrm{~ms}$ were removed from the analysis ( $4.34 \%$ of all trials). For visualization purposes, the saccade amplitudes shown in Figure 2A were smoothed using loess regression with a span of 15 trials. Raw saccade amplitudes across trial number (within the adaptation portion of each block) for each observer were fit to a parabolic function of the form:

$$
S_{a m p}=R n^{2}+D n+I
$$

where $S_{\text {amp }}$ represents the amplitude of the saccade, $n$ is the trial number, $R$ is the rate of change, and $D$ is the declivity of the parabola at the y-intercept (represented by $I$ ). Adaptation was summarized using the declivity parameter of the fit for the trials in the adaptation phase 
A
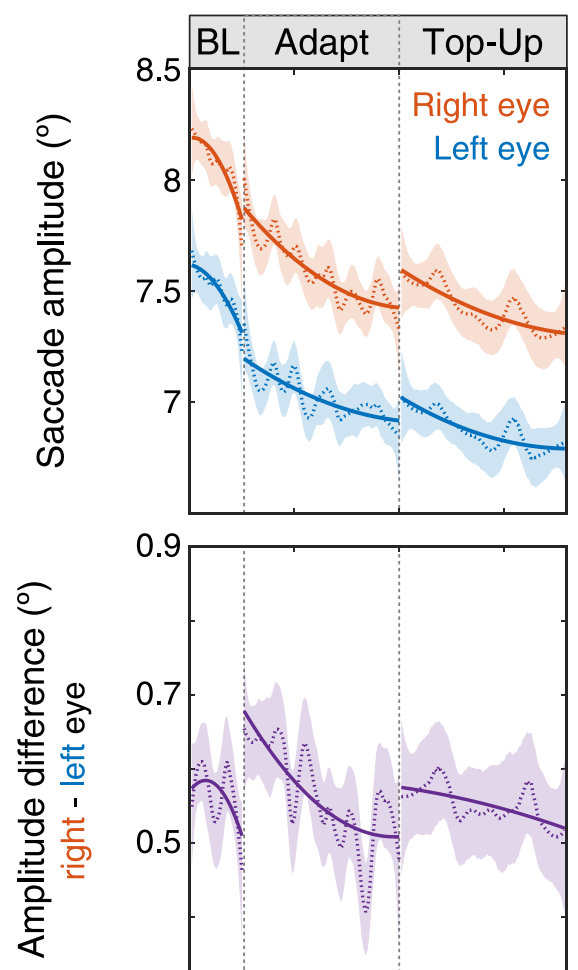

Conjugate Step

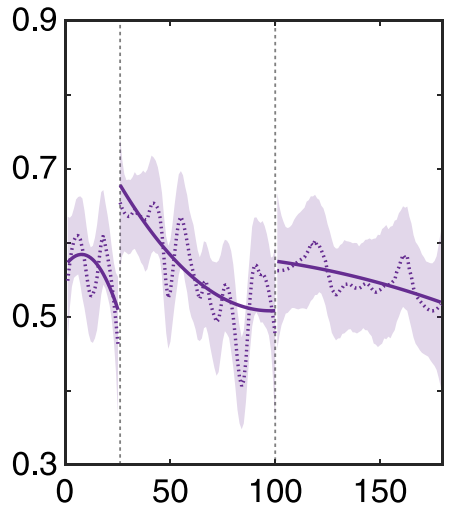

Dichoptic Step
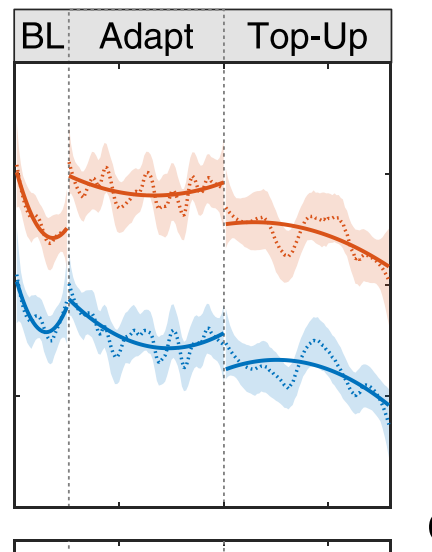

B

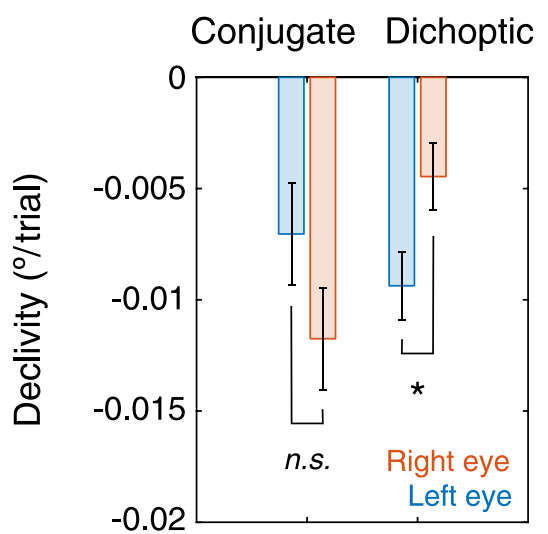

C

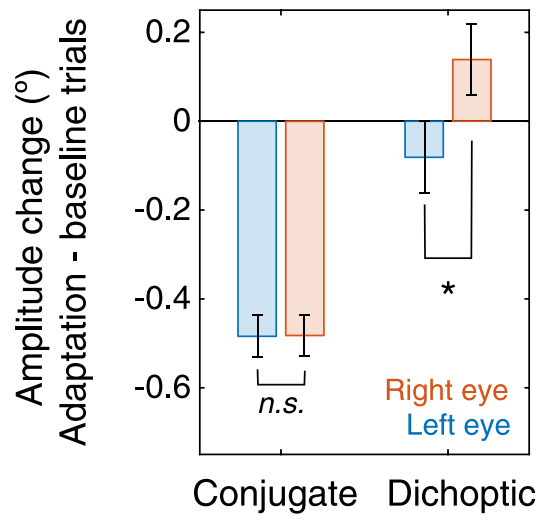

Trial number in block

Figure 2. Saccade amplitude results from Experiment 1. (A) Saccade amplitude as a function of trial number for each phase of the experiment ( $\mathrm{BL}=$ baseline), for the right and left eyes, shown in red and blue, respectively (upper panels), averaged across all six observers. For the trials labeled "top-up," the probe trials were not included in the analysis. The difference in amplitude (in the right eye minus the left eye) as a function of trial number is shown in the lower two panels. Shaded regions represent bootstrapped $95 \%$ confidence intervals. (B) Declivity parameter (degrees per trial) of the quadratic fits during the adaptation phase, calculated separately for each observer, and then averaged across observers. Negative values indicate a decrease in initial saccadic gain, and positive values indicate an increase in saccadic gain. (C) Amplitude change from baseline, calculated as the difference in saccade amplitude between baseline trials and adaptation trials for each eye and each condition. Error bars represent \pm 1 standard error of the difference between adjacent bars. Asterisks $\left({ }^{*}\right)$ denote significant pairwise comparisons $(p<0.05)$.

(trials 26-75), which represents the initial gain of the adaptation. Parameter estimates were averaged across observers and compared between conditions using a 2 (adaptation condition: conjugate vs. dichoptic) $\times$ 2 (eye: left vs. right) repeated-measures analysis of variance (ANOVA).

For the analysis of interocular divergence relative to saccade onset (Figure 3A), we calculated divergence as right minus left horizontal eye position over a window of -100 to $+500 \mathrm{~ms}$ relative to saccade onset on each trial. The reported divergence values are in degrees of visual angle. Trials with blinks, missing samples, or excessive differences in horizontal position between the two eyes $\left(>3^{\circ}\right.$; approximately twice the disparity) during this window were removed from the analysis (an additional $2.93 \%$ of all trials). As there were small amounts of $60 \mathrm{~Hz}$ noise produced by the active shutter glasses, gaze position samples were additionally filtered using a second-order Butterworth band-stop filter (2-Hz bandwidth) prior to averaging gaze samples across trials. Averages were then calculated for three types of trials: early adaptation (first 10 trials), late adaptation (last 10 trials), and probe trials (average of all probe trials). As each observer completed four blocks per adaptation condition, the interocular divergence for each adaptation condition was calculated over the average of 40 trials each for the early and late adaptation trials, and 160 trials for the probe trials. To isolate the effects of the adaptation manipulation on divergence, we subtracted the divergence values in the respective baseline trials for each condition, and calculated the divergence in the dichoptic minus the conjugate step conditions (Figure 3 ). This removed any transient divergence that was not specific to the 
A

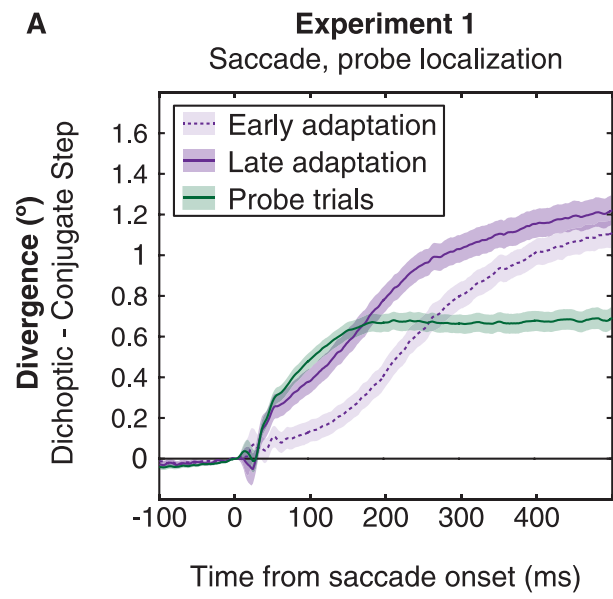

B Experiment 2
No saccade, probe localization

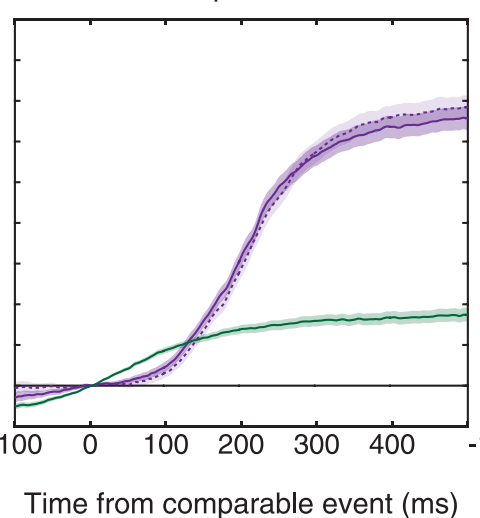

C Experiments 3A \& 3B Saccade, depth judgment

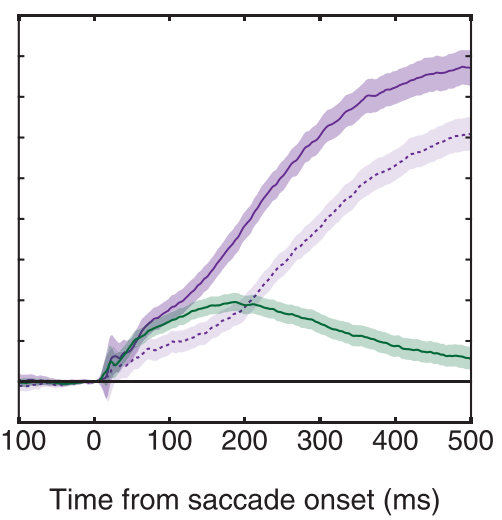

Figure 3. Interocular divergence (dichoptic minus conjugate step conditions) as a function of time relative to saccade onset for Experiments 1 to 3 ( $A-C$, respectively). As there was no saccade in Experiment 2, data points are plotted relative to the time of a comparable event in the trial sequence (stimulus step in adaptation trials, or simulated saccade in probe trials-see text). In panel $\mathrm{C}$, data are averaged across observers in Experiments 3A and 3B. The light and dark purple lines show averages for early and late adaptation trials, respectively. The green line shows the average across all probe trials. Shaded regions represent bootstrapped $95 \%$ confidence intervals for each condition.

adaptation (e.g., Collewijn, Erkelens, \& Steinman, 1997). When reported, $95 \%$ confidence intervals were estimated using a bootstrapping procedure (Efron \& Tibshirani, 1993). For each observer, we resampled individual trials within each condition (e.g., early adaptation) with replacement, and calculated the mean of the resampled trials. This procedure was repeated for 1000 iterations, and then averaged across participants to estimate the $95 \%$ confidence interval at the group level.

To validate our eye tracking results, we additionally measured the test-retest reliability of our adaptation effects between individual blocks within each adaptation condition. For each observer, we calculated the interocular difference in saccade amplitude across all trials within a single block (e.g., observer 1, conjugate step, block 1), and correlated all possible pairs of blocks within the same adaptation condition and observer (e.g., block 1 vs. block 2, block 1 vs. block 3). As shown in Supplementary Figure S1, we observed high test-retest reliability between all pairs of condition-matched blocks across all observers in Experiments 1 and 3, $r(106)=0.78, p<0.0001$. In addition, to ensure that our results are robust across different saccade detection criteria, we reanalyzed our results from Experiments 1 and 3 using the algorithm described in Engbert and Mergenthaler (2006; for details, see Supplementary Note 1 and Supplementary Figure S2), and observed similar results.

\section{Localization judgments}

As shown in Figure 4A, each observer's responses on probe trials were fit to a two-parameter logistic function
( $\alpha$ : horizontal shift, $\beta$ : slope) using maximum likelihood estimation. The position at which the second probe line appeared in the same location as the first probe (the point of subjective equality [PSE]) was calculated from the $50 \%$ point on the function.

The full set of data and materials are available on the Open Science Framework online: https://osf.io/fra2w/.

\section{Results}

\section{Saccadic adaptation}

The upper panels of Figure 2A show saccade amplitude for each eye across trial number during the adaptation period. In the conjugate step condition, we reproduced classic saccadic adaptation results (e.g., Deubel et al., 1986; McLaughlin, 1967). When there was a backward (inward) step in both eyes, saccade amplitudes decreased as a function of trial number during the adaptation phase in both the left eye and the right eye. In contrast, in the dichoptic step condition, saccade amplitudes decreased by a larger amount in the left eye than in the right eye. This can also be seen in the lower panels of Figure 2A, which show the difference in saccade amplitude between the two eyes (right eye minus left eye) across trial number. During the adaptation phase, the difference in saccade amplitude between the two eyes (right minus left) increased in the dichoptic step condition, consistent with the direction of the adapting step, and decreased in the conjugate step condition.

Together these changes in saccade amplitude are summarized in Figure 2B, in which the initial gain of 
the adaptation is represented by the declivity parameter (degrees per trial) of the parabolic fit to the raw saccade amplitudes over the adaptation phase. Positive values represent an initial increase in saccade amplitude, and negative values correspond to an initial decrease in amplitude. A 2 (adaptation condition: conjugate vs. dichoptic) x 2 (eye: left vs. right) repeated-measures ANOVA showed a significant interaction between adaptation condition and eye, $F(1,5)=8.76, p=0.032$. Pairwise comparisons of the declivity values between the two eyes indicated significantly higher declivity in the right eye compared with the left eye in the dichoptic step condition $t(5)=3.22, p=0.023$, consistent with the direction of the adaptation. There was no significant difference in the conjugate step condition, $t(5)=-2.05$, $p=0.096$. Neither the main effect of eye, nor the main effect of adaptation condition were significant, $F(1,5)<0.23, p>0.65$. We additionally verified the results shown in Figures $2 \mathrm{~A}$ and $2 \mathrm{~B}$ by comparing the difference in amplitude between the adaptation and baseline trials, independent of any fits to the data. As shown in Figure 2C, relative to the baseline trials, amplitudes in both eyes decreased in the conjugate step condition, and this change was similar between the two eyes $\left(-0.482^{\circ}\right.$ vs. $\left.-0.484^{\circ}, t(5)=0.038, p=0.97\right)$. In contrast, in the dichoptic step condition, relative to baseline, amplitudes during the adaptation phase were larger in the right eye and lower in the left eye $(+0.139$ vs. $-0.081, t(5)=2.73, p=0.041)$.

Figure $3 \mathrm{~A}$ shows the same changes in interocular divergence over the course of the adaptation period, showing the changes during the immediate postsaccadic interval. This plot shows the difference in interocular divergence between conditions, with divergence calculated as the difference in horizontal gaze position between the left and right eyes relative to saccade onset from -100 to $+500 \mathrm{~ms}$ (see Analyses). Over the full 500-ms postsaccadic window, we see significantly greater divergence in late compared with early adaptation trials, $t(5)=3.76, p=0.013$, which can be attributed to a shift in the speed at which the eyes diverge. Specifically, in early adaptation trials, the earliest time point at which we observed divergence significantly larger than zero was $150 \mathrm{~ms}, t(5)=2.89$, $p=0.034$, controlling for a false discovery rate of 0.05 across all time points using the Benjamini-Hochberg procedure. In contrast, in late adaptation trials, the earliest time point with divergence greater than zero was much earlier, only $36 \mathrm{~ms}$ after saccade onset, $t(5)=$ $2.99, p=0.030$.

Importantly, as shown in Figure 3A, during probe trials, we observed a retention of this adaptation effect at the time that the postsaccadic probe was shown. On probe trials, divergence is initially similar to the late adaptation trials during the period of the saccade $\left(0.213^{\circ}\right)$. Immediately following the end of the saccade, divergence values increased to $0.77^{\circ}$, plateauing at approximately $170 \mathrm{~ms}$ after saccade onset. We quantified this retention as the maximum divergence during probe trials divided by the maximum during late adaptation trials and found $63.1 \%$ retention of adaptation in the absence of a stimulus.

\section{Localization judgments}

The effects of adaptation retention were also seen in observers' location judgments during probe trials. The upper two panels of Figure 4A shows examples of psychometric functions for the dichoptic and conjugate step conditions, and the lower panel shows the PSEs for the group. The PSE values represent the position of the second line (test) that appears in the same position as the first line (the reference). A 2 (adaptation condition: conjugate vs. dichoptic) x 2 (eye: left vs. right) repeated-measures ANOVA on PSE values showed a significant main effect of adaptation condition, $F(1,5)$ $=17.49, p=0.009$, as well as a trending effect of eye, $F(1,5)=6.11, p=0.056$. These effects were qualified by a significant interaction between the adaptation condition and eye, $F(1,5)=13.95, p=0.014$. As shown in the bottom panel of Figure 4A, in the dichoptic step condition, PSEs were significantly different for left eye and right eye probes $\left(-1.92^{\circ}\right.$ vs. $-0.72^{\circ}$, respectively), $t(5)=3.25, p=0.023$. In other words, to appear in the same location, left-eye postsaccadic probes needed to be shifted to the left $\left(-1.92^{\circ}\right)$ to appear in the same location as the presaccadic reference. Right-eye probes needed to be shifted to the left, but by a much smaller amount $\left(-0.72^{\circ}\right)$.

For the conjugate step condition, PSEs were not significantly different between left and right eye probes $\left(-1.91^{\circ}\right.$ vs. $-1.88^{\circ}$, respectively) $t(5)=0.16, p=0.88$. The postsaccadic probe needed to be shifted to the left to appear in the same location as the presaccadic reference, and this result was not different for left- and right-eye probes, a finding consistent with Bahcall and Kowler (1999) and Collins et al. (2009). We note that, despite these differences between adaptation conditions, the PSEs for left- and right-eye probes in both conditions were negative. Although these negative values could be due to adaptation, they could also be produced by a general response bias across all conditions. To distinguish between these two possibilities, we collected additional baseline measurements of PSEs, without an adapting step, on four of the observers who completed Experiment 1 originally (see Supplementary Figure S3 and Supplementary Note 2). Consistent with previous work (Collins et al., 2009), the PSEs in the baseline data were closer to zero $\left(-0.65^{\circ}\right.$ vs. $-2.21^{\circ}$ for the baseline and the conjugate step conditions, respectively, $p<$ 0.001 , permutation test), indicating that the negative PSEs are largely due to adaptation.

Finally, we verified that observers were accurately performing the postsaccadic slant task on the 
A

Experiment 1

Saccade, probe localization

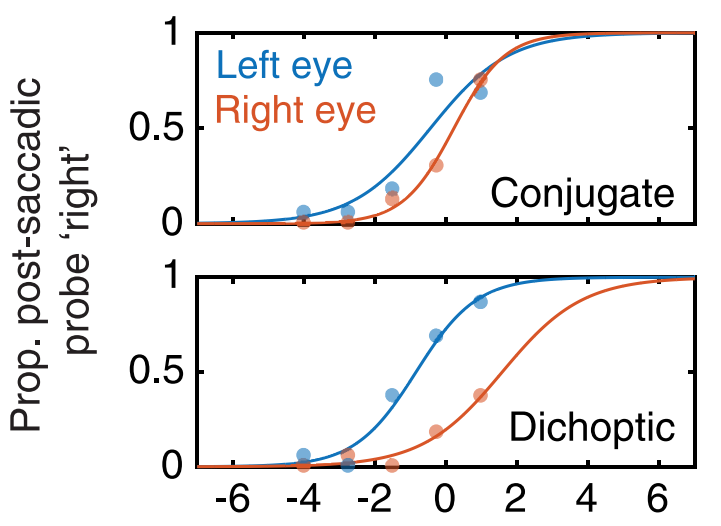

Post-saccadic probe location $\left({ }^{\circ}\right)$

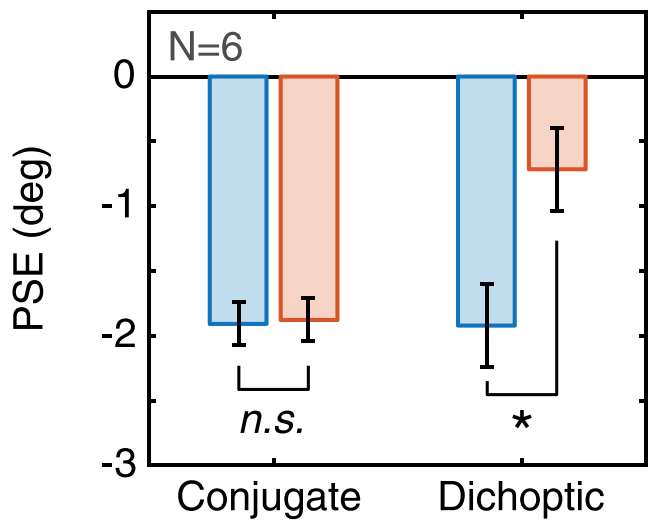

B

Experiment 2

No saccade, probe localization
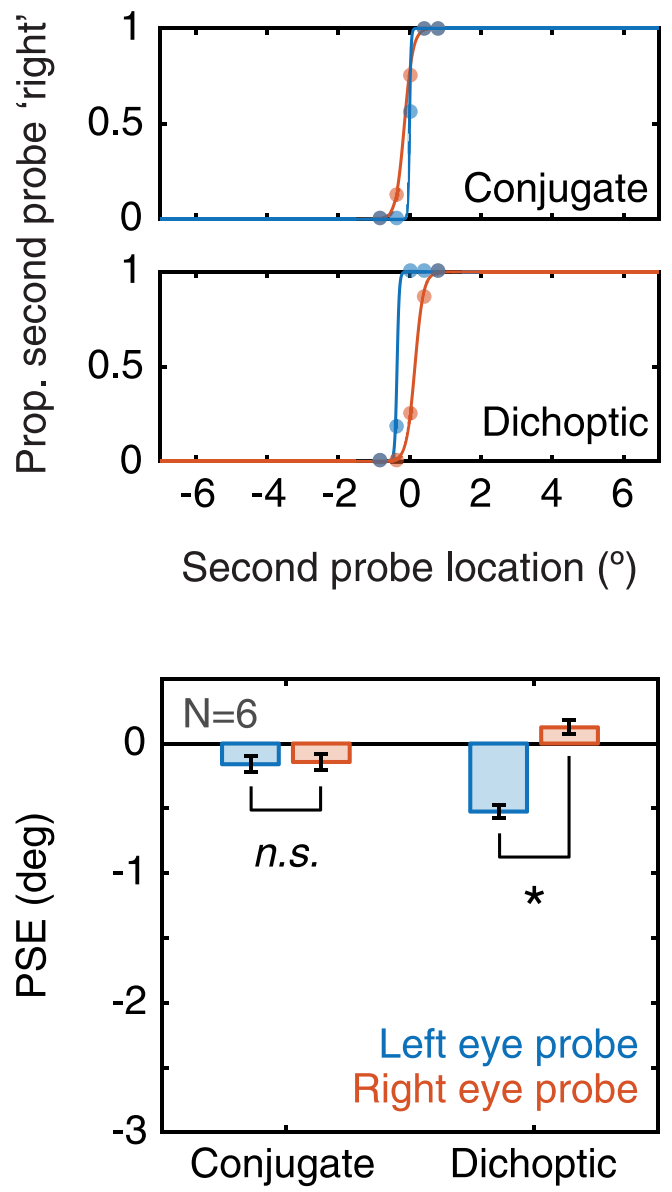

Figure 4. Behavioral results for Experiments 1 and 2 ( $A$ and $B$, respectively). The upper panels show example psychometric functions from one naive observer in each experiment, and the lower panels show the group average PSEs for each experiment. Red and blue data points in panels $A$ and $B$ represent right and left eye probes, respectively. Error bars represent \pm 1 standard error of the difference between adjacent bars (asterisks denote significant comparisons, $p<0.05$ ).

adaptation trials. Across participants, mean accuracy on the three-up, one-down staircase was $80.26 \%,(S D=$ $7.8 \%)$ with an average slant $(s)$ across reversals of 0.982 $(S D=0.02)$, corresponding to a maximum disparity of approximately 97.2 arcseconds. In addition, observers' performance was slightly lower in the dichoptic adaptation condition compared with the conjugate step condition (slant values of 0.979 vs. 0.985 ), but the difference did not reach significance, $t(5)=2.36, p=$ 0.065 .

\section{Discussion}

The results in Experiment 1 can be summarized as three main findings. First, our results provide evidence for unequal saccadic adaptation produced by dichoptic target steps. Consistent with Maiello et al. (2016), we show that adaptation in response to opposite target steps (Figure 1B) changes interocular divergence over a short timescale, measured as a change in the relative amplitude of saccades in the left and right eyes (Figure 2). However, we note that, in contrast to Maiello et al. (2016), in the dichoptic step condition, the changes over the course of the adaptation period were consistent with gain decreases for each eye. In other words, we report negative declivity values for each eye, rather than negative values for the left eye and positive values (i.e., an increase in gain) for the right eye. Although it is not clear why we observe this difference in our results, one possibility is that gain decreases might be induced more readily than gain increases. For example, previous work on saccadic adaptation has shown that, compared with gain decreases, gain increases follow a longer time course of adaptation and 
involve smaller amplitude changes (e.g., Deubel, 1991; Miller, Anstis, \& Templeton, 1981). Nevertheless, our results match the direction of the previously reported effects, with higher declivity values for the right eye compared with the left eye. In addition, when calculated relative to baseline, the mean amplitudes during the adaptation phase are consistent with the dichoptic step manipulation (i.e., a rightward step in the right eye and a leftward step in the left eye), as shown in Figure 2C.

Second, we measured how much divergence is retained following adaptation, when there is no sustained binocular stimulus. On probe trials, subjects executed a saccade to a brief monocular target, which disappeared by the time the eyes landed at that location. Here, there is no binocular information to drive vergence movements, but we nevertheless see a retention of $63.1 \%$ of the full divergence observed in the adaptation trials. This effect is distinct from previously reported recovery of saccade amplitude following adaptation (e.g., Maiello et al., 2016) as this shows how much the eyes diverge when there is no visual information to guide vergence movements (as opposed to a zero-disparity stimulus). To some extent, this retention indicates how much divergence is likely to be driven by saccade (rather than vergence) mechanisms. We also show that this effect cannot be attributed to a general tendency of observers to diverge their eyes when the saccade lands on a blank field; by calculating the difference between the dichoptic and conjugate adaptation conditions, we isolated an effect that was specific to adaptation in response to a dichoptic step.

Third, we report a perceptual effect following adaptation, in which postsaccadic probes need to be shifted in the same direction as the adapting step in the two eyes to be perceived in the same location as the presaccadic reference. We note that the PSE values for left- and right-eye probes were negative across all conditions, indicating a tendency to mislocalize postsaccadic test lines to the right of the presaccadic reference (i.e., in the same direction as the saccade). However, any differences between left- and right-eye probes appear to be superimposed on this general shift and are consistent with the direction of the adaptation in each condition. For example, the three conditions with a leftward adapting step (conjugate/right eye probe, conjugate/left eye probe, and dichoptic/left eye probe), all show similar negative PSEs (Figure 4A). In contrast, probes in the condition that had a rightward adapting step (dichoptic/right eye probe) needed to be shifted to the left by a much smaller amount to appear aligned with the presaccadic reference. These results show that when observers adapt unequally to opposite target steps in the two eyes, perception does not simultaneously compensate for the difference in saccade gain between the two eyes; instead, we observe perceptual changes, such that the apparent locations of the probes are shifted consistent with the direction of the adapting steps. If this shift was produced by a modification in the perceived location of the presaccadic target (Collins et al., 2009), this would suggest that saccadic adaptation can alter perceived space differently between the two eyes.

Finally, we note that, in addition to the unequal adaptation we observe in the dichoptic step condition, we see slightly unequal adaptation in the opposite direction during the adaptation phase of the conjugate step condition as well (lower-left panel in Figure 2A), even though the net disparity in this condition was zero. It is possible that this may be due to a small carryover effect across blocks of trials, which alternated between the conjugate and dichoptic adaptation conditions, even though we randomized whether the first block was conjugate or dichoptic. Unlike previous procedures (e.g., Maiello et al., 2016), we omitted recovery trials after the end of the adaptation period (which was necessary to keep the experiment at a reasonable duration for participants). Alternatively, there may be directional asymmetries in saccades (e.g., Collewijn, Erkelens, \& Steinman, 1988) that could produce slightly unequal adaptation between the two eyes in the conjugate step condition. Regardless, from Experiment 1, we can conclude that observers can adapt differentially to opposite target steps in the two eyes (Figure 2), and maintain this adaptation in the absence of a postsaccadic stimulus (Figure 3 ).

\section{Experiment 2: No saccade control}

Although the results in Experiment 1 demonstrate that observers show unequal adaptation in response to opposite target steps in the two eyes, one explanation of these findings could be based on vergence-based mechanisms, rather than independent monocular adaptation of saccades. Work in nonhuman primates, for example, has shown comparable saccadic adaptation based on binocular vergence mechanisms (Schultz \& Busettini, 2013). However, other work in humans has shown that it is possible to adapt saccades in depth without changing the vergence component of the eye movement (Chaturvedi \& Van Gisbergen, 1997).

In addition, it is possible that some of the divergence during probe trials reported in Experiment 1 could be an anticipatory or predictive response, in which participants may be accustomed to making the same vergence movement on every trial. Anticipatory or predictive changes in vergence movements are higher-level conditioned responses that occur when observers are shown repeated target steps in depth with a predictable direction (e.g., Alvarez, Bhavsar, Semmlow, Bergen, \& Pedrono, 2005; Kumar, Han, Garbutt, \& Leigh, 2002; Yuan, Semmlow, \& Munoz, 2000). Previous work has differentiated these types of 
higher-level, predictive mechanisms from adaptation by manipulating the predictability of the direction of the target steps (Alvarez et al., 2005). In this case, observers in Experiment 1 may be conditioned to make the same vergence movement on every trial, and as a result, diverge their eyes on probe trials even though there is no target step in depth.

It is unlikely that either of these factors could completely explain the results in Experiment 1, given the changes in saccade amplitude shown in Figures 2 and S1 (see also Maiello et al., 2016 for a discussion). Nevertheless, we can more directly isolate the relative contributions of saccades and vergence-related effects (e.g., adaptation or anticipatory responses) to the results we observed in Experiment 1 by measuring divergence and localization errors in the absence of a saccade. In Experiment 2, observers performed the same task, with the initial saccade removed from the trial sequence, and with all stimuli centered at the fovea. In the dichoptic step condition, observers made repeated vergence movements in response to a single target step the same size (total disparity of $1.6^{\circ}$ ) as that in Experiment 1. Aside from the absence of a saccade, the remaining stimulus parameters were matched as closely as possible between the two experiments. This provides an additional point of comparison, in which we can establish whether a saccade is necessary to see the same pattern of results observed in Experiment 1.

\section{Methods}

\section{Participants}

Six observers (four women; mean age, 29.8; age range, 24-36), including one author (AK), participated in Experiment 2. All observers had normal or corrected-to-normal vision and had stereoacuity of 60 arcseconds or better, measured with the Titmus stereo test. Three of the observers had previously completed Experiment 1.

\section{Stimuli and procedure}

The stimuli and procedure were similar to Experiment 1, with the first step removed from the adaptation sequences shown in Figure 1B, leaving only the second step. For both the adaptation and probe trials, the timing of the events in each trial, and all other parameters were intended to be otherwise identical to those in Experiment 1.

\section{Baseline and adaptation trials}

As in Experiment 1, observers were instructed to continuously fixate a colored square in the center of the black-and-white frame for 750 to $1250 \mathrm{~ms}$, which was centered in the middle of the display. The frame remained onscreen for a randomly selected interval that was intended to approximate saccade onset latency (see the simulated latency section later). After a 50-ms blank interval, the frame reappeared with a random set of black dots, which matched the parameters in Experiment 1. The first 25 trials of each block were baseline trials, in which the frame reappeared in the same location (i.e., at the fovea). In the adaptation and top-up adaptation trials, the frame was shifted to a different location. In the dichoptic step condition, the frame was shifted $0.8^{\circ}$ to the left in the left eye and $0.8^{\circ}$ to the right in the right eye, which was used to produce repeated vergence movements on each trial. In the conjugate step condition, the $0.8^{\circ}$ step was in the same direction in the two eyes: leftward on every trial for half of the observers, and rightward for the other half. After observers fixated the center of the frame continuously for 750 to $1250 \mathrm{~ms}$, it disappeared, and observers reported the slant of the dots as in Experiment 1. As expected, observers were able to accurately report the slant direction (mean performance of $83.4 \% ; S D=$ $1.7 \%)$. Across participants, the average slant $(s)$ across reversals was $0.987(S D=0.01)$, which corresponded to a maximum disparity of approximately 69.4 arcseconds.

\section{Probe trials}

After observers fixated the frame and fixation square for 750 to $1250 \mathrm{~ms}$, they were shown the first monocular probe (the reference line) for $100 \mathrm{~ms}$, centered at the fovea, in the same location as the frame. The second probe (the test line) appeared following a stimulus onset asynchrony equal to the trial's simulated saccade onset latency plus $250 \mathrm{~ms}$, to match the timing of the events in Experiment 1. The second probe was shown at one of five horizontal positions relative to the first probe, in linearly spaced steps from $-0.8^{\circ}$ to $+0.8^{\circ}$. As before, observers judged whether the second line appeared to the left or to the right of the first line.

\section{Simulated latency}

Although there was no initial $8^{\circ}$ step in Experiment 2, we approximated the timing of the events in Experiment 1 by assigning each trial to a simulated saccade onset latency. On each trial, the program selected a random number from an exponentially modified Gaussian distribution (ex-Gaussian) of the form:

$$
f(x)=\frac{1}{t} \exp \left(\frac{m-x}{t}+\frac{s^{2}}{2 t^{2}}\right) * .5\left(1+\left(\operatorname{erf}\left(\frac{\frac{x-m}{s}-\frac{s}{t}}{\sqrt{2}}\right)\right)\right)
$$

where the $\mu$ and $\sigma$ parameters control the mean and standard deviation of the Gaussian component, respectively, and $\tau$ controls the rightward skew of 
the distribution. The selected values for $\mu, \sigma$, and $\tau$ were $140 \mathrm{~ms}, 11 \mathrm{~ms}$, and $27 \mathrm{~ms}$, respectively, which were determined from the group average of the fits of the latency distributions in Experiment 1 (with the mean of the distribution at $167 \mathrm{~ms}$ ). Median saccade durations in Experiment 1 were relatively short, with little variance across observers $(44.3 \pm 3.5 \mathrm{~ms})$. We therefore used a 50-ms blank interval to approximate the duration of the saccade, as described earlier.

All procedures were otherwise identical to those in Experiment 1. Each observer completed 1440 trials across eight blocks (180 trials each), which alternated between the conjugate and dichoptic step conditions.

\section{Data analyses}

As there was no initial $\left(8^{\circ}\right)$ step in Experiment 2, observers did not make any large saccades. We therefore only analyzed interocular divergence, comparing early adaptation, late adaptation, and probe trials (Figure 3B). To facilitate comparisons to the results in Experiment 1, we calculated divergence near the time that the saccade would have normally occurred. For adaptation trials, this was the time of the frame's reappearance (i.e., the onset of the conjugate or dichoptic step). For probe trials, we used the time of the simulated saccade, which was in-between the two probes: on average, $167 \mathrm{~ms}$ after the onset of the first probe (randomly selected as described earlier), and exactly $250 \mathrm{~ms}$ before the onset of the second probe. For both trial types, the relevant event is plotted at $0 \mathrm{~ms}$, and as before, we report divergence from -100 to $+500 \mathrm{~ms}$ around the event. We removed $0.9 \%$ of trials from the analysis due to eye blinks or excessive divergence $\left(>3^{\circ}\right)$ within this time interval.

\section{Results and discussion}

We repeated the analyses from Experiment 1, calculating interocular divergence at comparable time points (Figure 3B), as well as the PSEs for observers' localization judgments in each condition (Figure 4B). Unlike Experiment 1, repeated vergence movements did not change between the first and last 10 adaptation trials, showing nearly identical changes when plotted relative to the time of the second step $(0 \mathrm{~ms})$. In the absence of a saccade, there were no significant differences in divergence between early and late trials, either averaged over the interval of 0 to $500 \mathrm{~ms}, t(5)$ $=0.018, p=0.987$, or at any of the individual time points, $t(5)<1.45 ; p>0.20$ (using a liberal $\alpha=0.05$, uncorrected for multiple comparisons). Therefore, we can conclude that saccade-based mechanisms, or the uncertainty of location perception around the time of saccades or in the peripheral visual field, are necessary for the changes between early and late trials observed in Experiment 1.

Although there was no difference in interocular divergence between early and late adaptation trials, we observed some divergence during probe trials. The maximum divergence over the same time interval ( 0 to $500 \mathrm{~ms}$ relative to stimulus onset) was $0.36^{\circ}$, and significantly larger than zero, $t(5)=7.81, p<$ 0.001 , suggesting that at least some of the retention effect in probe trials in Experiment 1 was due to repeated vergence movements (either adaptation or an anticipatory response), and not related to saccade execution. However, we note that this effect is $29.4 \%$ of the maximum divergence in adaptation trials, less than half the size of the retention effect observed in Experiment 1 (63.1\%; Figure 3A). In addition to the reduction in the size of this effect, it appears to be qualitatively different from the results in Figure 4A; divergence increases slowly over approximately 200 to $300 \mathrm{~ms}$, in contrast to the steep increase observed in Experiment 1.

Consistent with this smaller effect of adaptation on divergence in probe trials, we see a smaller effect on probe localization as well. Again, the direction of this effect was the same as in Experiment 1, but reduced to $53.7 \%$ of the localization effect observed in Experiment 1 (Figure 4B). Following the same analyses, a repeated-measures ANOVA on PSE values showed a significant interaction between adaptation condition and eye, $F(1,5)=61.5, p<0.001$. As shown in Figure 4B, in the dichoptic step condition, PSEs for left eye probes were lower (i.e., more leftward) than the PSEs right eye probes $\left(-0.53^{\circ}\right.$ vs. $0.12^{\circ}$, respectively), $t(5)=10.8, p<0.001$, consistent with the direction of the adapting step. As expected, PSEs in the conjugate step condition were not significantly different between the left and right eye probe conditions $\left(-0.16^{\circ}\right.$ vs. $-0.14^{\circ}$, respectively) $t(5)=0.27, p=0.80$.

These results indicate that both saccade and vergence-based mechanisms contribute to the results we observed in Experiment 1. When we eliminated saccades from the trial sequence, there was no difference in the time course of divergence in response to a single target step between early and late adaptation trials. One possible reason for the absence of an effect is that these analyses may not fully capture changes in the dynamics of the vergence response between early and late adaptation phase. For example, Yuan and Semmlow (2000) demonstrated that peak vergence velocity decreases after observers perform repetitive vergence movements in response to a single $4^{\circ}$ target step. Other work using a double-step paradigm has demonstrated that adaptive changes in vergence velocity can be induced without altering either the response latency or the final vergence angle (Takagi et al., 2001). Despite broadly similar vergence responses between early and 
late adaptation trials, we observed a low-amplitude vergence response during probe trials, in which there was no binocular stimulus presented. Identifying the underlying mechanism of this response would require further investigation, but one possibility is that this may be a high-level, predictive response, and we note that the amplitude of this response $\left(0.3^{\circ}-0.4^{\circ}\right)$ is similar to anticipatory or predictive vergence responses previously reported in the literature (Yuan et al., 2000).

Importantly, Experiment 1 demonstrated that eliminating the saccade from the trial sequence reduced the amplitude of divergence in probe trials, and the effect on localization errors by approximately half. Although it appears to be a vergence-based effect in probe trials (either due to adaptation or a predictive response), in which observers diverge their eyes when there is no binocular stimulus presented, this alone cannot account for the results that we saw in Experiment 1. Instead, a large portion of the effect that we observed in Experiment 1 is likely to be driven by saccades and possibly by the higher levels of perceptual uncertainty around the time of a saccade (e.g., Awater et al., 2005; Georg \& Lappe, 2009), and in the peripheral visual field to which saccades are directed. In a third and final experiment, we therefore evaluated a further possible perceptual effect of unequal saccadic adaptation produced by a dichoptic step by measuring observers' postsaccadic stereopsis thresholds.

\section{Experiment 3: Stereoacuity}

From Experiments 1 and 2, we can conclude that adaptation produces localization errors, as the adaptive oculomotor changes are not fully taken into account by the perceptual visual system. Do these effects extend to other types of perceptual judgment? An additional consequence that we might expect is a change in postsaccadic stereo performance. Following saccade completion, the eyes are diverged, and observer's gaze position is optimized for viewing an image with uncrossed disparity (i.e., behind the display). We predict that this would impair observers' ability to discriminate depth when they are presented with stereoscopic images that have net zero, or a near-zero disparity (i.e., no constant offset) following a saccade. In principle, this effect of a constant vergence error would be similar to the addition of a pedestal disparity (Blakemore, 1970; Krekling, 1974; McKee, Levi, \& Bowne, 1990; Ogle, 1953) or a prism-induced offset (Cole \& Boisvert, 1974; Ukwade, Bedell, \& Harwerth, 2003), both of which have been shown to elevate stereopsis thresholds. In summary, our prediction for Experiment 3 follows directly from the results of Experiments 1 and 2. If the perceptual visual system does not compensate for adaptive changes that are unequal between the two eyes, we would expect thresholds to be elevated following saccadic adaptation in response to a dichoptic step.

Measuring the effects of this adaptation procedure on stereo performance in normally sighted observers could also inform the development of similar procedures for improving stereopsis in individuals with vergence insufficiencies or some forms of strabismus. In principle, if this adaptation temporarily impairs stereo performance in normally sighted observers, it is possible that it may improve stereo thresholds in these patient populations if it induces correct alignment. Experiment 3 was designed to evaluate the first hypothesis here - that, in normally sighted individuals, we would see a transient elevation in stereopsis thresholds in the dichoptic step condition. In this experiment, observers completed adaptation procedures similar to those in Experiment 1. We predicted both saccadic adaptation (similar to that shown in Figure 2) and sustained divergence during probe trials in the absence of an adapting stimulus (Figure 3). On probe trials, at a similar time point following saccade onset, observers were shown a brief cluster of circles, and performed a four-alternative forced-choice (4AFC) depth discrimination task, in which they identified which circle had front depth (i.e., crossed disparity). As in Experiments 1 and 2, we compared performance to a control condition with a conjugate target step. To determine whether these effects were specific to the timing and duration of the stimulus in the depth discrimination task, we tested two different onset times (67 vs. $250 \mathrm{~ms}$ after saccade onset) and durations (175 vs. $150 \mathrm{~ms}$ ) across two experiments (Experiments 3A and 3B, respectively).

\section{Participants}

Twelve participants (eight women; mean age, 19.5; age range, 18-22) were recruited for Experiment 3A, and six were recruited for Experiment 3B (four women; mean age, 26.5; age range, 18-36), all with normal or corrected-to-normal vision and stereoacuity of 60 arcseconds or better, measured with the Titmus stereo test. Two of the participants in Experiment 3A did not perform significantly better than chance at the slant judgment across all slant trials ( $p>0.05$, binomial test), and another two had poor fits in the stereoacuity task (negative fitted slope or fitted threshold greater than $10,000 \mathrm{arcsec})$. These participants were removed from analysis, resulting in a final sample of eight participants in Experiment 3A (six women; mean age, 19.38; age range, 18-21), and six in Experiment 3B. None of the participants had previously completed Experiments 1 or 2 , and all were naive to the purpose of the experiment. 


\section{Stimuli and procedure}

The stimuli and procedure were similar to Experiment 1. The adaptation trials were identical to those in Experiment 1, and the probe trials measured stereoacuity instead of localization errors using the procedures described later. In Experiment 3A, observers completed two blocks of 196 trials each, one for each adaptation condition (dichoptic and conjugate target step conditions). In Experiment 3B, observers completed six blocks of 180 trials each (three in the conjugate step condition, and three in the dichoptic step condition). Each block consisted of 25 baseline trials, which had no second step, followed by 75 adaptation trials, and then 96 trials (Experiment 3A), or 80 trials (Experiment 3B) that alternated between probe trials and top-up adaptation trials, for a total of 48 (Experiment 3A) or 40 (Experiment 3B) probe trials per block. Half of the observers started with the conjugate step condition, and the other half started with the dichoptic condition. As before, adaptation conditions alternated (A-B-A-B) between blocks. Aside from these differences in the number of trials and the differences in probe timing listed in the following section, procedures were identical between Experiment 3A and 3B. As in Experiments 1 and 2, observers practiced both trial types ( 25 baseline trials and 10 probe trials) before starting the experiment. Performance in the slant discrimination task was similar to the other two experiments (accuracy of $79.4 \% ; S D=2.6 \%$, with the average of reversals equal to $0.985 ; S D=0.012$ ).

\section{Probe trials}

Stereopsis thresholds were estimated with a 4AFC task designed to be similar to the Titmus stereo test, which subjects were familiar with having completed the screening portion of the experiment. Each trial started with an initial fixation interval in which observers were shown an empty frame and fixation point, centered $4^{\circ}$ to the left of screen center. After continuously fixating this target for 750 to $1250 \mathrm{~ms}$, the frame and fixation point shifted $8^{\circ}$ to the right and disappeared after $100 \mathrm{~ms}$. Observers were instructed to saccade to this target and maintain their gaze at that side of the screen. At $250 \mathrm{~ms}$ (Experiment 3A) or $67 \mathrm{~ms}$ (Experiment 3B) after saccade onset, observers were shown a cluster of four circular sinusoidal gratings centered in the same location as the saccade target, and arranged in a diamond configuration (see Figure 5A), with a center-to-center distance of $4^{\circ}$ between opposite pairs. Each grating had a spatial frequency of 1.5 cycles per degree and was shown inside a hard circular aperture $\left(2^{\circ}\right.$ diameter) at $100 \%$ Michelson contrast. Within each cluster, one randomly selected circle had crossed disparity (in front of the screen), and the remaining three circles had uncrossed disparity (behind the screen). Crossed and uncrossed disparities were produced by equal but opposite horizontal shifts of the left- and right-eye images by half the full disparity, and the absolute disparity was the same for each circle. The cluster of circles disappeared after $175 \mathrm{~ms}$ (Experiment 3A) or $150 \mathrm{~ms}$ (Experiment 3B), and observers reported the location of the front-depth circle using one of the four arrow keys (left, right, up, or down) on the keyboard. This short duration was determined from pilot testing, and was intended to give observers sufficient time to perform the task accurately, while minimizing observers' ability to complete vergence movements in response to the stimulus, which have typical latencies of approximately 160 to $180 \mathrm{~ms}$ (e.g., Rashbass \& Westheimer, 1961).

The disparity on each trial was controlled using a one-up, three-down staircase procedure. The starting level of the staircase in both the conjugate and dichoptic adaptation conditions was calculated using the pretest procedure described later. The initial step size was 30 arcseconds, which was reduced by $25 \%$ every three reversals, and the program constrained the disparity values so that they could not fall outside the range of zero to 1000 arcseconds. To improve efficiency, the staircase followed a one-up, one-down rule until the first reversal. Observers were given feedback on the accuracy of their responses on every trial, identical to the feedback provided for the slant judgment. A green or red fixation square on the next trial indicated a correct or incorrect response, respectively.

\section{Stereoacuity pretest}

To further improve the efficiency of the staircase in the face of the broad distribution of stereoacuity in the healthy population (Hess, To, Zhou, Wang, \& Cooperstock, 2015), the initial disparity level for both adaptation conditions was established by a pretest procedure, which observers completed at the beginning of the experiment. Observers completed one block consisting only of 48 probe trials, following the procedures described in the previous section. The initial disparity value for the staircase was 200 arcseconds for all observers. Following completion of this pretest, we calculated the average of all reversal points, and used this value as the starting point for the staircase in each adaptation condition. Across observers, these estimates ranged from 27.9 to 519.6 arcseconds, with an average of 155.8 arcseconds.

\section{Analyses}

The eye tracking data were analyzed using the same procedures described in Experiment 1. Using the same exclusion criteria as the previous experiments, across 
A

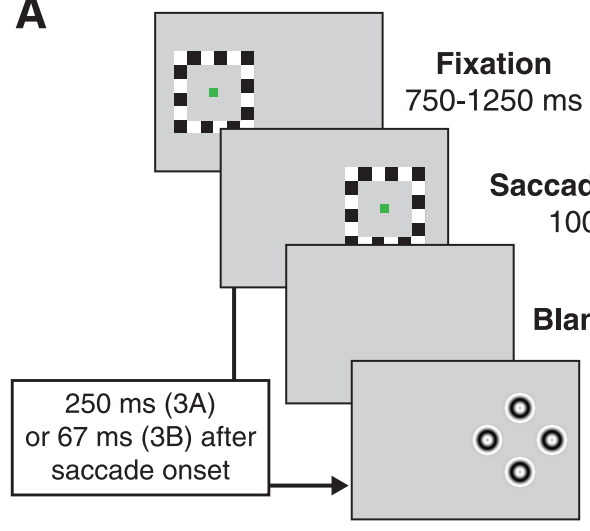

B

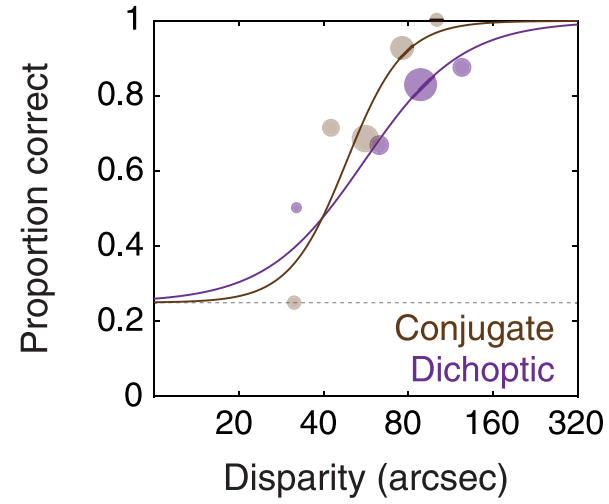

Disparity (arcsec)

Identify the front-depth circle:

Stereoacuity test

$175 \mathrm{~ms}(3 \mathrm{~A})$ or $150 \mathrm{~ms}$ (3B) duration

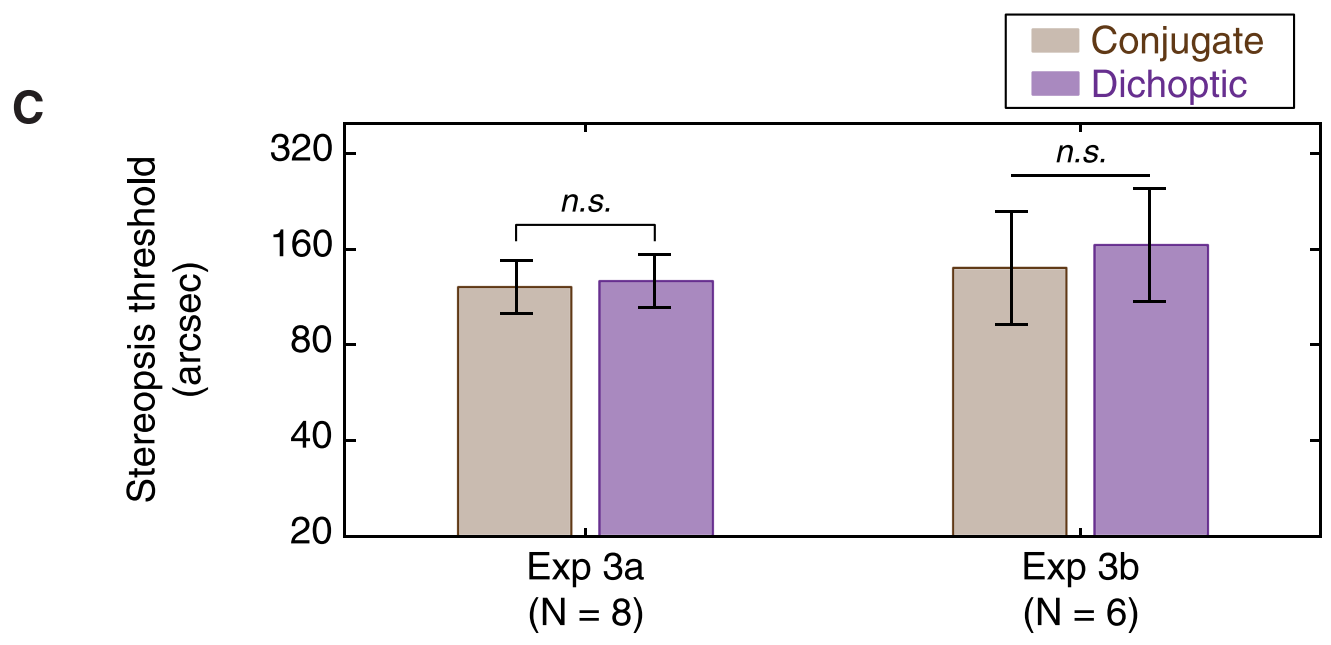

Figure 5. Procedure and behavioral results for Experiments $3 \mathrm{~A}$ and $3 \mathrm{~B}$. (A) Probe trials in Experiments $3 \mathrm{~A}$ and $3 \mathrm{~B}$ were used to measure stereopsis thresholds in each adaptation condition. Following saccade onset and a brief blank interval, observers were shown a cluster of four circular sinusoidal gratings (175 or $150 \mathrm{~ms}$ ) and identified the front-depth circle in a 4AFC task. Baseline and adaptation trials in these experiments followed the same procedures as those in Experiment 1. (B) Stereopsis thresholds were estimated from psychometric fits to observers' data in each adaptation condition, as shown for one representative naive observer. (C) Group averages of stereopsis thresholds for Experiments 3A and 3B showed no significant difference in stereopsis thresholds between the two adaptation conditions in either experiment. Error bars represent \pm 1 standard error of the difference between adjacent bars.

Experiments $3 \mathrm{~A}$ and $3 \mathrm{~B}$, we removed $6.66 \%$ of the trials, which did not have a saccade within the amplitude and latency bounds, and an additional $1.75 \%$ of trials due to eye blinks or excessive divergence $\left(>3^{\circ}\right)$ during the postsaccadic interval. One observer had a large proportion of trials $(29.3 \%)$ meeting these exclusion criteria and was removed from the saccade analyses. We note that the number trials in which we measured divergence for early adaptation, late adaptation, and probe trials was somewhat lower than our previous experiments (respectively, up to 10, 10, and 48 trials per observer per condition in Experiment $3 \mathrm{~A}$, and 30, 30, and 120 trials in Experiment 3B), resulting in slightly greater variability in our divergence estimates. As with the location judgments in Experiments 1 and
2 , stereo performance was also fit to a two-parameter logistic function, with a floor of $25 \%$, corresponding to chance performance in a 4AFC task. Disparity levels were converted to $\log _{10}$ units prior to curve fitting and averaging across subjects, and stereoacuity was calculated as the disparity level corresponding to $80 \%$ performance. In Experiment 3B, individual threshold estimates for each block were averaged within each condition for each subject.

\section{Results and discussion}

Following the analysis procedures from Experiment 1, we observed similar saccadic adaptation effects, showing 
unequal saccadic adaptation between the two eyes in the dichoptic step condition. Supplementary Figure S4 shows saccade amplitudes across Experiments 3A and 3B, similar to Figure 2. As before, we observed significantly higher declivity in the right eye compared with the left eye in the dichoptic step condition, $t(12)=$ $3.15, p=0.008$. There was no significant difference in the conjugate step condition, $t(12)=0.75, p=0.47$. In addition, the changes in amplitude, measured relative to baseline, followed the same direction as the results in Experiment 1. In the conjugate step condition, we observed similar amplitude decreases in both eyes $\left(-0.38^{\circ}\right.$ vs. $\left.-0.36^{\circ}\right), t(12)=0.47, p=0.64$. In the dichoptic step condition, we observed a significant difference between the amplitude changes in the two eyes $\left(+0.03^{\circ}\right.$ vs. $\left.-0.17^{\circ}\right), t(12)=3.36, p=0.006$, which was consistent with the direction of the adapting step.

Figure $3 \mathrm{C}$ shows interocular divergence near the time of the saccade for Experiments $3 \mathrm{~A}$ and $3 \mathrm{~B}$ combined. Following the analysis procedures from the previous two experiments, we see significantly larger divergence in late compared with early adaptation trials over the full 500-ms postsaccadic window, $t(12)=7.08, p<$ 0.001 . Consistent with this result, the first time point with a divergence value significantly larger than zero (after controlling for a false discovery rate of 0.05 ) was $70 \mathrm{~ms}, t(12)=2.41, p=0.033$, in early adaptation trials. In contrast, the same time point in late adaptation trials was closer to the time of saccade onset $(22 \mathrm{~ms}$ after onset), $t(12)=2.30, p=0.04$. Despite these similarities to Experiment 1, on probe trials, we observed a different pattern of divergence after saccade completion, with the values initially increasing to $0.44^{\circ}$ (mean of individual subject maxima), peaking at approximately $190 \mathrm{~ms}$ after saccade onset, and then decreasing to $0.11^{\circ}$. Although divergence was significantly larger than zero over the full 0 - to $500-\mathrm{ms}$ window during probe trials, $t(12)=5.40, p=0.0002$, it was reduced compared with Experiment 1, and the size of the retention effect (maximum divergence in probe trials divided by late adaptation trials) was $0.44^{\circ}$, or $28.3 \%$.

Consistent with this smaller divergence in probe trials, there was also no significant difference in stereoacuity between the two adaptation conditions in Experiment 3A, with mean thresholds of 121.4 and 126.7 arcseconds in the conjugate and dichoptic step conditions, respectively (Figure $5 \mathrm{C}$ ) $, t(7)=0.22, p=$ 0.83 . The absence of an effect here was independent of our manipulation of the timing parameters; we also observed no difference in stereopsis thresholds in Experiment 3B (139.5 vs. 164.5 arcseconds), $t(5)$ $=0.40, p=0.70$, in which probe onset was closer to the time of the saccade. We note that these thresholds are slightly higher than expected, given observers' performance on the Titmus stereo test during the screening procedure (60 arcseconds or less for all participants). It is possible that the brief stimulus duration contributed to this elevation in thresholds, consistent with previous work showing impaired stereo performance with brief durations (Harwerth \& Rawlings, 1977; Ogle \& Weil, 1958). Importantly, the stimulus duration and other stimulus parameters were matched between the conjugate and dichoptic step conditions and cannot account for the lack of an effect on stereopsis thresholds.

What accounts for this reduction in divergence during probe trials, compared with the results in Experiment 1? One possibility is that unequal adaptation in response to a dichoptic step wears off more rapidly because observers are shown a binocular stimulus with a near-zero disparity (i.e., no constant offset) on every other trial. In other words, for the last portion of each block, the postsaccadic disparity information in the probe trials may undo some of the adaptation effect (i.e., an effect similar to interleaving adaptation and recovery trials). This could also explain the lack of an effect of adaptation on stereopsis thresholds; if there is a smaller vergence error, any elevation in observers' thresholds would be smaller, and more difficult to detect in an experiment. Although we cannot definitively conclude that there is no effect of unequal saccadic adaptation on stereopsis thresholds, it appears that any effect, if it exists, is likely to be smaller and less robust than the effect on localization, as observed in Experiment 1.

\section{General discussion}

To briefly summarize the effects of adaptation on eye movements, in Experiment 1, our results are consistent with previous work demonstrating unequal saccadic adaptation produced by a dichoptic step (Maiello et al., 2016). This adaptation is likely to be driven by a combination of monocular and binocular mechanisms, and consistent with these previous findings, we observed changes in monocular saccade amplitudes (Figure 2), as well as binocular vergence movements following saccade completion (Figure 3). In Experiment 2, we showed that removing the initial target step (i.e., the saccade) from the sequence of events eliminated the changes in vergence movements between early and late adaptation trials. This demonstrates that repeated vergence movements alone are insufficient to produce the changes observed during the adaptation phase. We also measured the extent to which adaptation is retained in probe trials when observers are not provided with any binocular information following a saccade. In Experiment 1, we showed that divergence in these trials is approximately $63 \%$ of the maximum during adaptation. Experiment 2 demonstrated that although there is some component of this retention that is due 
to vergence-based mechanisms, it cannot account for the entire magnitude or the temporal profile of the divergence we observed. When measuring the perceptual consequences of adaptation, we observed an effect on localization, consistent with previous results (Bahcall \& Kowler, 1999), but no significant effect on stereopsis thresholds. To some extent, the size of any perceptual effect may be related to the size of the retention on probe trials. We observed the largest perceptual effect in Experiment 1, which also had the largest divergence during probe trials. The results in Experiments 2 and 3 both showed reduced divergence in probe trials, and also reduced or absent perceptual effects. The localization errors we observed in Experiment 1 have several implications for our understanding of perceptual stability in three-dimensional space. First, these results suggest that the visual system does not automatically account for the unequal changes in saccade amplitude when spatial positions are compared across saccades. In order for pre- and postsaccadic probes to appear in the same location, postsaccadic targets needed to be shifted in the same direction as the adapting steps, which were in opposite directions in the two eyes. Further experiments would be necessary to establish whether these spatial localization dissociations would be present during steady fixation, without any executed saccade, and whether offsets would be present uniformly across the visual field or spatially localized at the saccade endpoint. Errors that are uniform or that occur in the absence of a saccade might suggest a more general change in spatial representations following adaptation (e.g., Zimmermann \& Lappe, 2010). Although the literature generally assumes that the visual system relies on a single spatial map for oculomotor control in the two eyes (e.g., Anderson et al., 1994; Fox, Fox, Raichle, \& Burde, 1985), results showing independent recalibration of saccade amplitudes, along with independent changes to spatial representations in the two eyes following adaptation, might provide some evidence for separable maps for oculomotor control in the two eyes.

A further implication of the position judgment data emerges from the fact that targets are mislocalized differently in the two eyes. In principle, using binocular (rather than monocular) probes should result in a shift in apparent depth when comparing pre- and postsaccadic targets, such that observers would misplace postsaccadic targets in depth. However, when we performed a pilot experiment to test observers' ability to compare the relative depths of pre- and postsaccadic targets, observers had difficulty performing the task reliably. It is likely that the targets' brief duration, as well as their spatial and temporal separation, introduced additional noise to observers' judgments. Moreover, the use of binocular targets during probe trials reduced the magnitude of divergence. As we note in Experiment 3, adaptation may be more difficult to maintain when top-up adaptation trials are interleaved with binocular test images that have no double-step offset.

\section{Conclusions}

If long-term adaptation to sensory and motor changes (e.g., corrective lenses, surgery, normal aging) relies on mechanisms similar to saccadic adaptation (e.g., Lemij \& Collewijn, 1991; Scudder et al., 1998), it is important to consider how these changes impact perception. The present results indicate that perceptual changes may not automatically follow oculomotor changes, and that combined oculomotor and sensory adaptation may be needed to change both (for clinical implications, see Zhou, Wang, Feng, Wang, \& Hess, 2017). Further work will be necessary to determine whether the localization errors would persist over longer time scales, and whether similar dichoptic perceptual errors are observed in individuals who begin wearing corrective lenses or undergo surgery. Measuring localization biases and precision in these instances would provide insight into the circumstances under which the visual system compensates for sensory and motor changes, if at all, and more broadly, how the visual system maintains stability across saccades in three-dimensional space.

Keywords: binocular vision, localization, saccadic adaptation, vergence

\section{Acknowledgments}

The authors thank Oishi Hawlader for her assistance in developing these experiments as part of the Research Science Institute (RSI), and Kelly Choi for her assistance with data collection.

$$
\begin{aligned}
& \text { Supported by R01 EY029713 (PJB) and F32 } \\
& \text { EY028814 (AK). }
\end{aligned}
$$

Commercial relationships: none. Corresponding author: Anna Kosovicheva. Email: anna.kosov@gmail.com. Address: Department of Psychology, Northeastern University, Boston, MA, USA.

\section{References}

Alahyane, N., Salemme, R., Urquizar, C., Cotti, J., Guillaume, A., \& Vercher, J. L., ...Pélisson, D. (2007). Oculomotor plasticity: Are mechanisms 
of adaptation for reactive and voluntary saccades separate? Brain Research, 1135(1), 107-121, http://doi.org/10.1016/j.brainres.2006.11.077.

Albano, J. E., \& Marrero, J. A. (1995). Binocular interactions in rapid saccadic adaptation. Vision Research, 35(23-24), 3439-3450, http://doi.org/10.1016/0042-6989(95)00079-T.

Alvarez, T. L., Bhavsar, M., Semmlow, J. L., Bergen, M. T., \& Pedrono, C. (2005). Short-term predictive changes in the dynamics of disparity vergence eye movements. Journal of Vision, 5(7):4, 640-649, http://doi.org/10.1167/5.7.4.

Anderson, T. J., Jenkins, I. H., Brooks, D. J., Hawken, M. B., Frackowiak, R. S. J., \& Kennard, C. (1994). Cortical control of saccades and fixation in man A PET study. Brain, 117(5), 1073-1084, http://doi.org/10.1093/brain/117.5.1073.

Averbuch-Heller, L., Lewis, R. F., \& Zee, D. S. (1999). Disconjugate adaptation of saccades: Contribution of binocular and monocular mechanisms. Vision Research, 39(2), 341-352, http://doi.org/10.1016/S0042-6989(98)00156-4.

Awater, H., Burr, D., Lappe, M., Morrone, M. C., \& Goldberg, M. E. (2005). The effect of saccadic adaptation on the perception of visual space. Journal of Neurophysiology, 93, 3605-3614, http://doi.org/10.1152/jn.01013.2003.

Bahcall, D. O., \& Kowler, E. (1999). Illusory shifts in visual direction accompany adaptation of saccadic eye movements. Nature, 400(6747), 864-866, http://doi.org/10.1038/23693.

Blakemore, C. (1970). The range and scope of binocular depth perception in man. Journal of Physiology, 211(3), 599-622.

Brainard, D. H. (1997). The Psychophysics Toolbox. Spatial Vision, 10(4), 433-436, http://doi.org/10.1163/156856897X00357.

Bridgeman, B., Hendry, D., \& Stark, L. (1975). Failure to detect displacement of the visual world during saccadic eye movements. Vision Research, 15(6), 719-722, http://doi.org/10.1016/0042-6989(75) 90290-4.

Bruno, A., \& Morrone, M. C. (2007). Influence of saccadic adaptation on spatial localization: Comparison of verbal and pointing reports. Journal of Vision, 7(5):16, 1-13, http://doi.org/10.1167/7.5. 16.Introduction.

Chaturvedi, V., \& Van Gisbergen, J. A. M. (1997). Specificity of saccadic adaptation in threedimensional space. Vision Research, 37(10), 1367-1382, http://doi.org/10.1016/S0042-6989(96) 00266-0.

Clark, R. A., \& Demer, J. L. (2002). Effect of aging on human rectus extraocular muscle paths demonstrated by magnetic resonance imaging. American Journal of Ophthalmology, 134(6), 872-878, http://doi.org/10.1016/S0002-9394(02) 01695-1.

Cole, R. G., \& Boisvert, R. P. (1974). Effect of fixation disparity on stereo-acuity. Optometry and Vision Science, 51(3), 206-213, http: //doi.org/10.1097/00006324-197403000-00006.

Collewijn, H., Erkelens, C. J., \& Steinman, R. M. (1988). Binocular co-ordination of human horizontal saccadic eye movements. The Journal of Physiology, 404(1), 157-182, http://doi.org/10.1113/jphysiol.1988.sp017284.

Collewijn, H., Erkelens, C., \& Steinman, R. (1997). Trajectories of the human binocular fixation point during conjugate and non-conjugate gaze-shifts. Vision Research, 37(8), 1049-1069.

Collins, T., Rolfs, M., Deubel, H., \& Cavanagh, P. (2009). Post-saccadic location judgments reveal remapping of saccade targets to non-foveal locations. Journal of Vision, 9(5):29, 1-9, http://doi.org/10.1167/9.5.29.

Cornelissen, F. W., Peters, E. M., \& Palmer, J. (2002). The Eyelink Toolbox: Eye tracking with MATLAB and the Psychophysics Toolbox. Behavior Research Methods, Instruments, \& Computers, 34(4), 613-617, http://doi.org/10.3758/BF03195489.

Coubard, O. A. (2013). Saccade and vergence eye movements: A review of motor and premotor commands. European Journal of Neuroscience, 38(10), 3384-3397, http: //doi.org/10.1111/ejn.12356.

Deubel, H. (1991). Adaptive control of saccade metrics. Presbyopia Research, 93-100, http: //doi.org/10.1007/978-1-4757-2131-7_11.

Deubel, H., Wolf, W., \& Hauske, G. (1986). Adaptive gain control of saccadic eye movements. Human Neurobiology, 5(4), 245-253.

Efron, B., \& Tibshirani, R. J. (1993). An introduction to the bootstrap. London: Chapman \& Hall.

Engbert, R., \& Mergenthaler, K. (2006). Microsaccades are triggered by low retinal image slip. Proceedings of the National Academy of Sciences of the United States of America, 103(18), 7192-7197, http://doi.org/10.1073/pnas.0509557103.

Erkelens, C. J., Collewijn, H., \& Steinman, R. M. (1989). Asymmetrical adaptation of human saccades to anisometropic spectacles. Investigative Ophthalmology and Visual Science, 30(6), 1132-1145.

Fox, P. T., Fox, J. M., Raichle, M. E., \& Burde, R. M. (1985). The role of cerebral cortex in the generation of voluntary saccades: A positron emission tomographic study. 
Journal of Neurophysiology, 54(2), 348-369, http://doi.org/10.1152/jn.1985.54.2.348.

Georg, K., \& Lappe, M. (2009). Effects of saccadic adaptation on visual localization before and during saccades. Experimental Brain Research, 192(1), 9-23, http://doi.org/10.1007/s00221-008-1546-y.

Harwerth, R. S., \& Rawlings, S. C. (1977). Viewing time and stereoscopic threshold with random-dot stereograms. American Journal of Optometry and Physiological Optics, 54(7), 452-457, http://doi.org/10.1097/00006324-19770700000004.

Hering, E. (1868). Die lehre vom binokularen sehen. Leipzig: Wilhelm Engelmann.

Hess, R. F., To, L., Zhou, J., Wang, G., \& Cooperstock, J. R. (2015). Stereo vision: The haves and have-nots. I-Perception, 6(3), 1-5, http://doi.org/10.1177/2041669515593028.

Hopp, J. J., \& Fuchs, A. F. (2004). The characteristics and neuronal substrate of saccadic eye movement plasticity. Progress in Neurobiology, 72(1), 27-53, http://doi.org/10.1016/j.pneurobio.2003.12.002.

King, W. M. (2011). Binocular coordination of eye movements: Hering's Law of equal innervation or uniocular control? European Journal of Neuroscience, 33(11), 2139-2146, http://doi.org/10.1111/j.1460-9568.2011.07695.x.

Kleiner, M., Brainard, D., \& Pelli, D. G. (2007). What's new in Psychtoolbox-3? Perception 36 ECVP Abstract Supplement, 36(14), 1.

Krekling, S. (1974). Stereoscopic threshold within the stereoscopic range in central vision. Optometry and Vision Science, 51(9), 626-634, http://doi.org/10.1097/00006324-197409000-00002.

Kumar, A. N., Han, Y., Garbutt, S., \& Leigh, R. J. (2002). Properties of anticipatory vergence responses. Investigative Ophthalmology and Visual Science, 43(8), 2626-2632.

Lemij, H. G., \& Collewijn, H. (1991). Long-term nonconjugate adaptation of human saccades to anisometropic spectacles. Vision Research, 31(11), 1939-1954, http://doi.org/10.1016/0042-6989(91) 90189-C.

Mack, A., Fendrich, R., \& Pleune, J. (1978). Adaptation to an altered relation between retinal image displacements and saccadic eye movements. Vision Research, 18(10), 1321-1327, http://doi.org/10.1016/0042-6989(78)90222-5.

Maiello, G., Harrison, W. J., \& Bex, P. J. (2016). Monocular and binocular contributions to oculomotor plasticity. Scientific Reports, 6, 1-7, http://doi.org/10.1038/srep31861.
McKee, S. P., Levi, D. M., \& Bowne, S. F. (1990). The imprecision of stereopsis. Vision Research, 30(11), 1763-1779, http://doi.org/10.1016/0042-6989(90) 90158-H.

McLaughlin, S. C. (1967). Parametric adjustment in saccadic eye movements. Perception \& Psychophysics, 2(8), 359-362, http://doi.org/10. 3758/BF03210071.

Miller, J. M., Anstis, T., \& Templeton, W. B. (1981). Saccadic plasticity: Parametric adaptive control by retinal feedback. Journal of Experimental Psychology: Human Perception and Performance, 7(2), 356-366, http://doi.org/10.1037/0096-1523.7. 2.356 .

Moidell, B. G., \& Bedell, H. E. (1988). Changes in oculocentric visual direction induced by the recalibration of saccades. Vision Research, 28(2), 329-336, http://doi.org/10.1016/0042-6989(88) 90161-7.

Ogle, K. N. (1953). Precision and validity of stereoscopic depth perception from double images. Journal of the Optical Society of America, 43(10), 906-913, http://doi.org/10.1364/josa.43.000906.

Ogle, K. N., \& Weil, M. P. (1958). Stereoscopic vision and the duration of the stimulus. $A M A$ Archives of Ophthalmology, 59(1), 4-17, http: //doi.org/10.1001/archopht.1958.00940020028002.

Panouillères, M., Weiss, T., Urquizar, C., Salemme, R., Munoz, D. P., \& Pélisson, D. (2009). Behavioral evidence of separate adaptation mechanisms controlling saccade amplitude lengthening and shortening. Journal of Neurophysiology, 101(3), 1550-1559, http://doi.org/10.1152/jn.90988.2008.

Pélisson, D., Alahyane, N., Panouillères, M., \& Tilikete, C. (2010). Sensorimotor adaptation of saccadic eye movements. Neuroscience and Biobehavioral Reviews, 34(8), 1103-1120, http://doi.org/10.1016/j.neubiorev.2009.12.010.

Pelli, D. G. (1997). The VideoToolbox software for visual psychophysics: Transforming numbers into movies. Spatial Vision, 10(4), 437-442, http://doi.org/10.1163/156856897X00366.

Rashbass, C., \& Westheimer, G. (1961). Disjunctive eye movements. The Journal of Physiology, 159(2), 339360, http://doi.org/10.1113/jphysiol.1961.sp006812.

Ross, J., Morrone, M. C., \& Burr, D. C. (1997). Compression of visual space before saccades. Nature, 386, 598-601.

Schultz, K. P., \& Busettini, C. (2013). Shortterm saccadic adaptation in the macaque monkey: A binocular mechanism. Journal of Neurophysiology, 109(2), 518-545, http: //doi.org/10.1152/jn.01013.2011. 
Scudder, C. A., Batourina, E. Y., \& Tunder, G. S. (1998). Comparison of two methods of producing adaptation of saccade size and implications for the site of plasticity. Journal of Neurophysiology, 79(2), 704-715, http://doi.org/10.1152/jn.1998.79.2.704.

Shaw, R. B., Katzel, E. B., Koltz, P. F., Yaremchuk, M. J., Girotto, J. A., Kahn, D. M., ... Langstein, H. N. (2011). Aging of the facial skeleton: Aesthetic implications and rejuvenation strategies. Plastic and Reconstructive Surgery, 127(1), 374-383, http://doi.org/10.1097/PRS.0b013e3181f95b2d.

Stampe, D. M. (1993). Heuristic filtering and reliable calibration methods for video-based pupil-tracking systems. Behavior Research Methods, Instruments, \& Computers, 25(2), 137-142, http://doi.org/10.3758/BF03204486.

Takagi, M., Oyamada, H., Abe, H., Zee, D. S., Hasebe, H., Miki, A., .. . Bando, T. (2001). Adaptive changes in dynamic properties of human disparity-induced vergence. Investigative Ophthalmology and Visual Science, 42(7), 1479-1486.

Ukwade, M. T., Bedell, H. E., \& Harwerth, R. S. (2003). Stereopsis is perturbed by vergence error. Vision Research, 43(2), 181-193, http://doi.org/10.1016/S0042-6989(02)00408-X.
Volkmann, F. C. (1962). Vision during voluntary saccadic eye movements. Journal of the Optical Society of America, 52(5), 571-578, http://doi.org/10.1364/josa.52.000571.

von Helmholtz, H. (1867). Handbuch der physiologischen optik. Leipzig: L. Vos.

Yuan, W., \& Semmlow, J. L. (2000). The influence of repetitive eye movements on vergence performance. Vision Research, 40(22), 3089-3098, http://doi.org/10.1016/S0042-6989(00)00162-0.

Yuan, W., Semmlow, J. L., \& Munoz, P. (2000). Effects of prediction on timing and dynamics of vergence eye movements. Ophthalmic and Physiological Optics, 20(4), 298-305, http://doi.org/10.1016/S0275-5408(99)00093-9.

Zhou, J., Wang, Y., Feng, L., Wang, J., \& Hess, R. F. (2017). Straightening the eyes doesn't rebalance the brain. Frontiers in Human Neuroscience, 11, 1-8, http://doi.org/10.3389/fnhum.2017.00453.

Zimmermann, E., \& Lappe, M. (2010). Motor signals in visual localization. Journal of Vision, 10(6):2, 1-11, http://doi.org/10.1167/10.6.2. 